\title{
Evaluation of Urinary Arsenic as an Indicator of Exposure to Residents of Tarkwa, Ghana
}

\author{
K. A. Asante ${ }^{1,2 *}$, T. Agusa ${ }^{1}$, R. Kubota ${ }^{1}$, A. Subramanian 1 , O. D. Ansa-Asare ${ }^{2}$, C. A. Biney ${ }^{2}$ and S. Tanabe ${ }^{1}$ \\ ${ }^{1}$ Center for Marine Environmental Studies (CMES), Ehime University, Bunkyo-cho 2-5, Matsuyama, Ehime, 790- \\ 8577, Japan \\ ${ }^{2}$ CSIR-Water Research Institute, P.O. Box AH 38, Achimota, Accra, Ghana \\ *Corresponding author; E-mail: kaasante@chemist.com
}

\begin{abstract}
In Ghana, mining plays a significant role in the economic recovery programme. However, the gains are achieved at the cost of environmental and human health. For many years, the extraction of gold involved roasting which released airborne particles and large quantities of arsenic (As). Sampling for this study was conducted in March 2004 to assess the contamination status of trace elements, especially As, in water and mine workers in Tarkwa, which has nearly a century of gold mining history. Water and human urine samples were collected from Tarkwa, in addition to control samples taken from Accra, the capital of Ghana. Arsenic excretion was assessed in the first morning void urine. Concentrations of As and $\mathrm{Mn}$ in some water samples from Tarkwa were above the WHO drinking water guidelines. A potential health risk of As and $\mathrm{Mn}$ is a concern for the people consuming the contaminated water in this area. Levels of trace elements in water from control site were low compared to levels from Tarkwa. The mean urinary As concentration of $260 \mu \mathrm{gl}^{-1}$ from the study area was comparable to those in Asendemic areas of the world. This indicates relatively high degree of human exposure to As in Tarkwa, Ghana. Relatively low levels of As in water and no significant difference of As concentrations in urine between Tarkwa and Accra may suggest the presence of other sources of As contamination in Ghana, possibly food. This is the first study reporting 23 trace elements in human urine samples from a mining town in Ghana.
\end{abstract}

\section{Introduction}

Arsenic (As) is an ubiquitous element found in several forms in foods and environmental media such as soil, air and water. The predominant form in drinking water is inorganic As, which is both highly toxic and readily bioavailable (Valenzuela et al., 2005). Chronic ingestion of inorganic As-contaminated drinking water is, therefore, considered the major pathway behind the risk to human health. It was estimated that 200 million people worldwide are at risk from health effects associated with high concentrations of As in their drinking water (NRC, 2001). Large scale human exposure to As through contaminated groundwater is a serious health threat in many Asian and Latin American countries (Watanabe et al., 2001). With the exception of some studies in Taiwan (Tseng, 1977; Hsueh et al., 1998), attention has only recently been given to the epidemiological and human toxicological aspects of this contamination.

In Ghana, mining constitutes a great portion of the country's gross national product, and is the bedrock of the country's economic recovery programme. Ghana is a major gold producing country in West Africa and gold accounts for the second largest foreign exchange earner after cocoa. However, the gains are achieved at the cost of environmental and human health. Exploitation of gold puts immense stress on air, water, soil, and vegetation, and also, frequently, poses hazards to human health (Amonoo-Neizer \& Amekor, 1993). For many years, the extraction of gold involved roasting which released airborne particles and large quantities of As (14-19 tons) daily in Obuasi (Amonoo-Neizer \& Amekor, 1993). The main prospects in Ghana occur at Obuasi, Tarkwa, Prestea, Bibiani, Bogoso and Dunkwa, with the gold occurring in close association with sulphide minerals, especially arsenopyrite and pyrite (Smedley, 1996).

In Obuasi, which is the most important mining area in Ghana, arsenic contamination of food and water has been linked with gold-mining activities. Smedley et al. (1996) reported concentrations of up to $350 \mu \mathrm{g} \mathrm{l}^{-1}$ in stream waters affected directly by mining pollution in Obuasi area, and Amonoo-Neizer \& Amekor (1993) reported high As concentrations in food crops $\left(0.12-70.5 \mathrm{mg} \mathrm{kg}^{-1}\right)$ also in Obuasi. At least $10 \%$ of Ghana's rural borehole wells have As concentrations exceeding $10 \mu \mathrm{g} \mathrm{l}^{-1}$ (Mead, 2005). Earlier studies reported that mining operations have resulted in significant, localized surface water, soil, plant, some food items, human hair and atmospheric As pollution in Obuasi (Smedley et al., 1996; Smedley \& Kinniburgh, 2002; Amonoo-Neizer \& Busari, 1980; Amasa, 1975; Amonoo-Neizer, 1980). 
The determination of toxic elements in urine is an important clinical screening procedure and has become a matter of wide interest owing to the toxicity of these elements and their influence in controlling the course of biological processes (Horng et al., 2002). Urine is easily sampled and, for instance, urinary $\mathrm{Pb}$ had been employed in occupational health as a noninvasive surrogate (Alessio \& Foa, 1983) especially when subjects were reluctant to allow blood drawing. The arsenic content of urine is generally regarded as the most reliable indicator of recent exposure to inorganic As and is used as the main biomarker of exposure (Mushak \& Crocetti, 1995).

Arsenic tends not to accumulate in the body but is readily excreted via the kidneys (Vahter \& Lind, 1986). Urine is, therefore, a good indicator of environmental As exposure. Urinary As has long been used as a measure of exposure to the element, although, in the early days, the analytical methodology was not sensitive enough to detect the natural background concentration found in all samples (Lai et al., 2004). In addition, the urinary profiles of inorganic As metabolites have recently been used in epidemiological studies to assess the capacity of exposed individuals to methylate inorganic As (Valenzuela et al., 2005).

Humans are exposed to As in many different ways; by ingestion of contaminated water and food, by inhalation of As-containing dust or volatile As compounds, or by ingestion of As-containing preparations (Luh et al., 1973). There is evidence that As (III), once absorbed, may be oxidized to As (V) and or methylated in the human body. When inorganic arsenicals are ingested, urine forms the main route of elimination and the metabolites of inorganic As; dimethy-larsinic acid (DMA) and monomethylarsonic acid (MMA) are the predominant species in urine (Lagerkvist et al., 1988). Braman \& Foreback (1973) were the first to report on the identification of As species in

human urine and Smedley et al. (1996) reported a mean urinary total As concentration of $260 \mu \mathrm{g}^{-1}$ from two sample villages, Wumase and Kofikurom, in the Obuasi area of Ghana.

Previous studies (Golow \& Adzei, 2002; Adimado \& Baah, 2002; Golow et al., 1996, Amonoo-Neizer \& Amekor, 1993; Amasa, 1975; Smedley, 1996, Smedley et al., 1996) have reported mainly on As, Hg and a few trace elements in environmental samples from Ghana. The aim of the study was to assess the extent of trace elements exposure, especially As to mine workers and inhabitants of Tarkwa. This is the first comprehensive study reporting 23 trace element levels in human urine samples from a mining town in Ghana.

\section{Materials and methods}

\section{Study area}

Tarkwa and its surrounding villages (Fig. 1), which constitute the focus of the study, have nearly a century of gold mining history. The area experiences one of the heaviest and most frequent rains in the country. The choice of this region is important for a number of reasons. Apart from the operations of AngloGold Ashanti Company (formerly Ashanti Goldfields Company Ltd, AGC) at Obuasi in the Ashanti Region, most of the country's gold, diamond, and manganese and bauxite mines are located in this region. Secondly, the area has the largest concentration of mines in a single district on the continent, and, virtually, all the mining companies operating surface mines in the area are international, with mining concessions and operations in several other African countries (Akabzaa, 2000). 


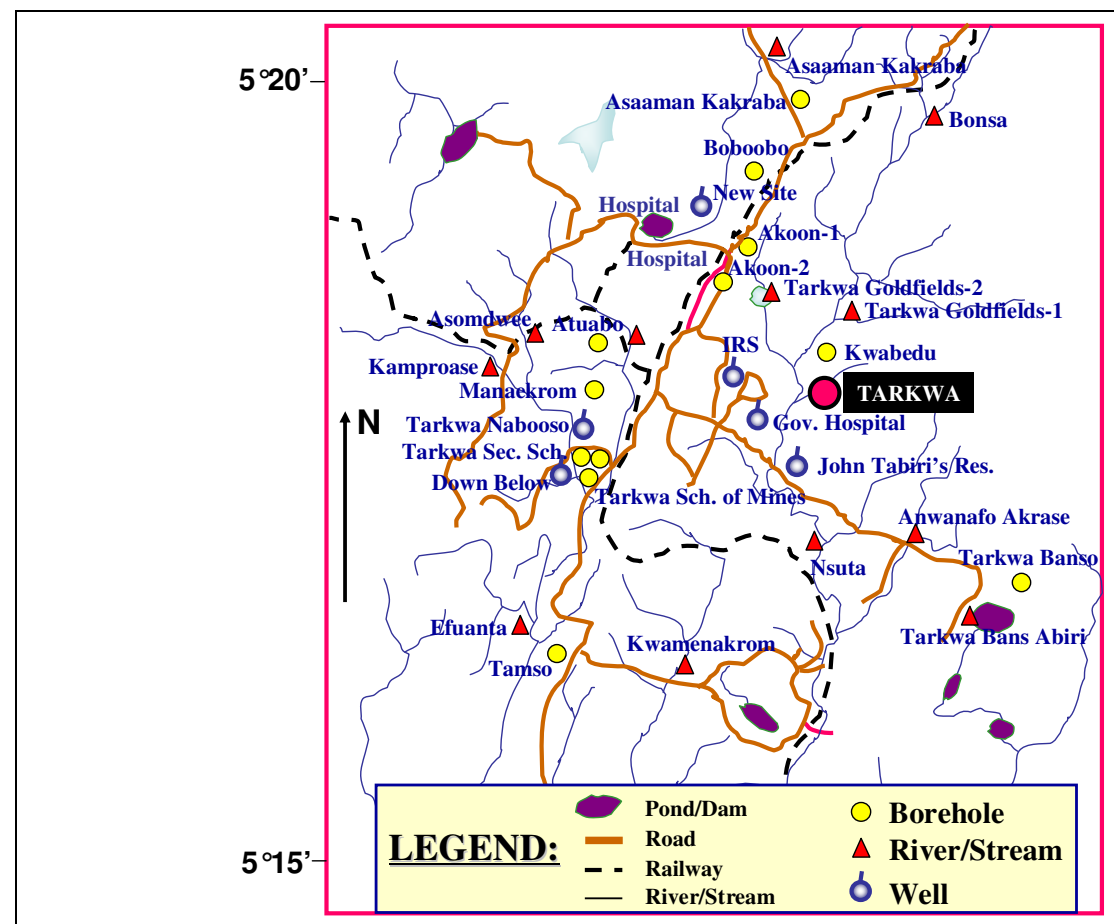

Figure 1. Map showing sampling locations of Tarkwa and surrounding areas.

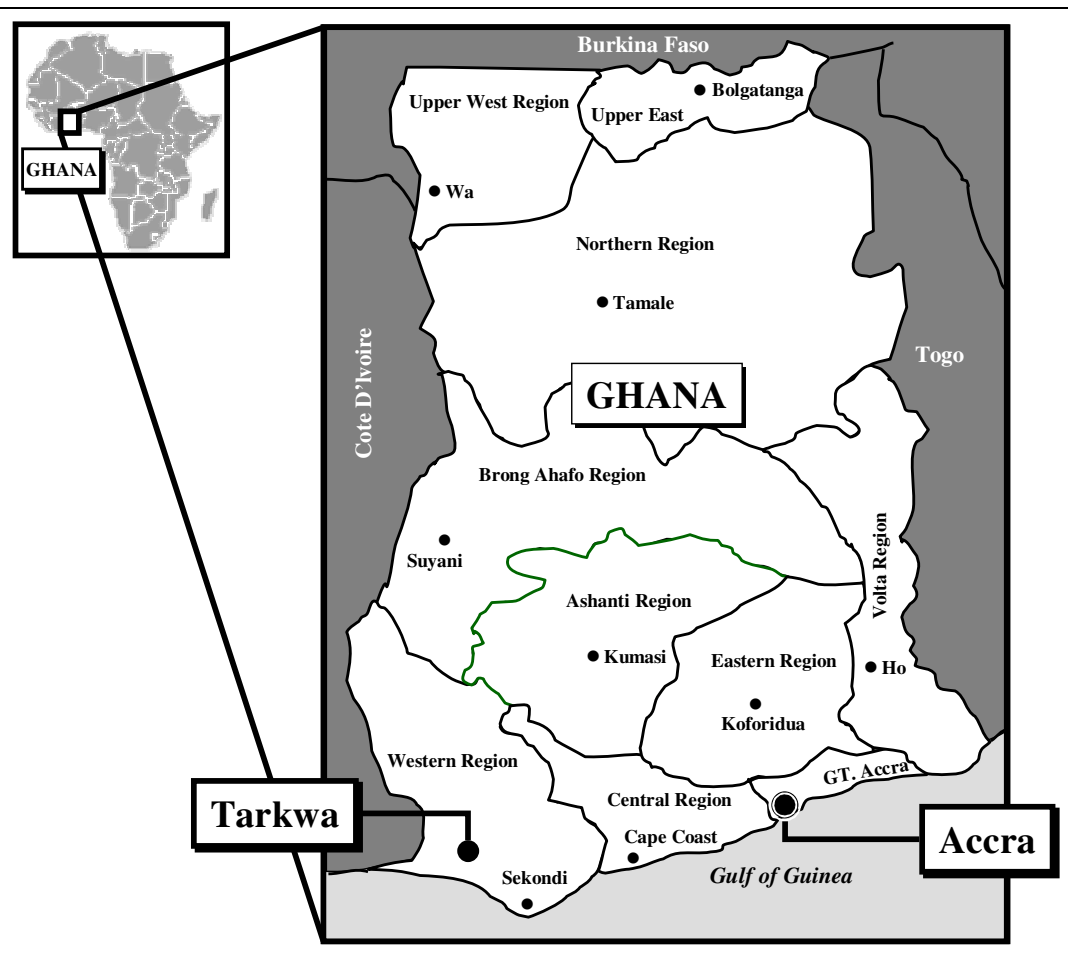

Figure 1a. Map of Ghana showing study area (Tarkwa) and control site (Accra). 
The study area is part of the Wassa West District of the Western Region with Tarkwa as its district capital. The district is defined by latitudes $4^{\circ} \mathrm{N}$ and $5^{\circ} 40^{\prime} \mathrm{N}$, and longitudes $1^{\circ} 45^{\prime} \mathrm{W}$ and $2^{\circ} 10^{\prime} \mathrm{W}$. The area falls within the equatorial climatic zone. Rainfall peaks during two periods, April-June and October-November, with an annual mean rainfall of $1933 \mathrm{~mm}$ (Akabzaa, 2000). Relative humidity for the area ranges from 83\% to $91 \%$ with temperature varying between $28{ }^{\circ} \mathrm{C}$ and $33{ }^{\circ} \mathrm{C}$ (Kuma \& Younger, 2001). The Tarkwa District is part of an extensive drainage basin known as the Ankobra basin, comprising the Ankobra river and its tributaries.

\section{Samples}

Thirty water and 32 human urine samples were collected from Tarkwa and its environment in March 2004, by a team from the Council for Scientific and Industrial Research-Water Research Institute (CSIR-WRI), Accra, Ghana. The water samples were from boreholes $(n=12)$, wells $(n=6)$ and rivers/streams $(n=12)$. The borehole samples were taken from the standpipes after $30 \mathrm{~min}$ of flushing. The wells were selected based on water demand of the surrounding communities as well as the scarcity of water from the public supplies. All well water samples were taken with a water sampler. The streams/rivers were sampled under base flow conditions about 20-30 cm depth from the surface. The $p \mathrm{H}$ values of the water samples were measured in-situ. One control water sample was also taken from a stand pipe located on the premises of the CSIR-WRI in Accra, the capital city of Ghana, and a distance of about $317 \mathrm{~km}$ from Tarkwa (Fig. 1a).

Spot urine samples $(n=32)$ were obtained from patients at the Tarkwa Government Hospital and Aba Hospital (a private hospital), with the assistance of nurses. They included mine workers (14 males and 1 female) and non-mine workers ( 7 males and 10 females). The ages of the mine workers ranged from 26 to 59 years while that of the nonmine workers varied between 19 and 51 years. As control, urine samples were obtained from four male workers (25-27 years) of the CSIR-WRI in Accra, where no mining occurs. The informed consent was obtained from all the donors in an ethical manner. All the water and urine samples were collected in polyethylene bottles which were prescreened in Japan. The samples were not subjected to any chemical treatment. They were stored in cold boxes in the field and, on return to the laboratory in Accra, kept in a deep freezer. The frozen samples were air-freighted to the Center for Marine Environmental Studies (CMES) of the Ehime University in Japan, and were kept in an Environmental Specimen Bank $(e s-B A N K)$ at $-20^{\circ} \mathrm{C}$ until chemical analyses.

\section{Chemical analyses}

The water samples were acidified with concentrated sulphuric acid for As and nitric acid for the other trace elements. Milli-Q water acidified with sulphuric or nitric acid was used as a blank. Concentrations of trace elements (V, Cr, Mn, Co, Cu, Zn, Ga, Rb, Sr, Mo, Ag, Cd, In, Sn, Sb, Cs, Ba, Tl, Pb and Bi) were measured by an inductively-coupled plasma mass spectrometer (ICP-MS, HP-4500, Hewlett-Packard, Avondale, PA, USA) (Agusa et al., 2006). Matrix effects and instrumental drift in the ICP-MS measurements were corrected by the internal standard method with indium as the internal standard. Concentrations of $\mathrm{Hg}$ and $\mathrm{Se}$ were determined by cold vapour-atomic absorption spectrometry (CV-AAS; Model HG-3000, Sanso, Tsukubu, Japan) and hydride generation atomic absorption spectrometry (HG-AAS; Model HFS-3, Hitachi, Tokyo, Japan), respectively. Arsenic determination was by hydride generation-atomic absorption spectrophotometer (HG-AAS) using a Shimadzu HVG1 hydride generation system coupled to a Shimadzu AA680 AAS (Shimadzu, Kyoto, Japan).

Urine samples were digested by heating with a mixture of sulphuric acid, nitric acid and perchloric acid for As determination carried out by HG-AAS. For other trace elements, a closed vessel microwave system (Ethos D, Milestone S.r.l., Sorisole, BG, Italy) was used for acid digestion of human urine samples. The digestion programme applied was $2 \mathrm{~min}$ at $250 \mathrm{~W}, 3 \mathrm{~min}$ at $0 \mathrm{~W}, 5 \mathrm{~min}$ at $250 \mathrm{~W}, 5 \mathrm{~min}$ at $400 \mathrm{~W}, 5 \mathrm{~min}$ at $500 \mathrm{~W}, 10 \mathrm{~min}$ at $400 \mathrm{~W}$ and 5 min for ventilation, respectively.

\section{Statistical analysis}

Statistical analysis was performed using SPSS (version 12.0, SPSS Inc., Chicago, Il, USA) for windows. MannWhitney $U$-test was used for the detection of differences in trace element concentrations in urine between males and females, mine workers and non-mine workers, as well as people in mine region (Tarkwa) and in control site (Accra). The Spearman rank correlation was used to examine the correlation between age and trace element concentrations in human urine samples. For samples having concentrations below the detection limits of the trace elements, one 
half of the value of the respective detection limit was substituted and used in statistical analysis. A probability value of $P<0.05$ was considered as statistically significant in this study.

\section{Water samples}

\section{Results and discussion}

Groundwaters (wells and boreholes) in this study area recorded $p \mathrm{H}$ values varying between 4.02 and 6.90 (Table 1). Most groundwaters in southern Ghana, in particular, fall within that range and the reasons are likely related to local geology. Kuma \& Younger (2001) reported that the $p \mathrm{H}$ of the soils in the Tarkwa area varies from very acidic to moderately acidic, a condition attributed to the tropical climate and lush vegetation. Arsenic concentrations in the groundwaters ranged from $<0.1 \mu \mathrm{g} \mathrm{l}^{-1}$ to $4 \mu \mathrm{g} \mathrm{l}^{-1}$ (Table 1). On the other hand, As concentrations in rivers/streams which had relatively high $p \mathrm{H}$ values between 5.82 and 7.85 were from $<1 \mu \mathrm{g} \mathrm{l}^{-1}$ to $73 \mu \mathrm{g} \mathrm{l}^{-1}$ (mean value of $19 \mu \mathrm{g} \mathrm{l}^{-1}$ ). About $33 \%$ of the river water samples in this study exceeded the World Health Organization (WHO) guideline value of $10 \mu \mathrm{g} \mathrm{l}^{-1}$ for As in drinking water (WHO, 2004) (Fig. 2). The highest As concentration of $73 \mu \mathrm{g} \mathrm{l^{-1 }}$ was from a lake-like water body and downstream of the study area from which the inhabitants draw their domestic water.

TABLE 1

Trace element concentrations $(\mu \mathrm{g} / \mathrm{L})$ in water samples from the study area (Tarkwa) and control site (Accra).

\begin{tabular}{|c|c|c|c|c|c|c|c|c|c|c|c|c|c|}
\hline Source & Type & $p H$ & $V$ & $\mathrm{Cr}$ & $M n$ & Co & $C u$ & $Z n$ & $G a$ & As & $\mathrm{Se}$ & $R b$ & $\mathrm{Sr}$ \\
\hline Tarkwa School of Mines & Borehole & 5.70 & 3.2 & 2.1 & 2.2 & 0.02 & 11.1 & 6.88 & 0.08 & 2.0 & $<0.1$ & 0.79 & 83.3 \\
\hline Tarkwa Secondary School (1) & Borehole & 6.90 & 3.4 & 0.37 & 4970 & 3.3 & 1.79 & 3.96 & 20.9 & 0.5 & $<0.1$ & 1.81 & 1320 \\
\hline Tarkwa Secondary School (2) & Borehole & 6.75 & 1.1 & 0.12 & 1450 & 0.94 & 1.87 & 3.64 & 0.95 & 1.0 & $<0.1$ & 0.85 & 657 \\
\hline Akoon (1) & Borehole & 4.04 & 0.01 & 0.03 & 1000 & 8.9 & 7.11 & 21.2 & 1.54 & 0.5 & $<0.1$ & 2.18 & 38.3 \\
\hline Akoon (2) & Borehole & 5.85 & 1.5 & 1.5 & 634 & 0.13 & 4.08 & 5.79 & 1.18 & 3.0 & $<0.1$ & 1.59 & 522 \\
\hline Atuabo & Borehole & 5.49 & 0.18 & 0.46 & 229 & 0.87 & 1.19 & 24.5 & 0.96 & 1.0 & $<0.1$ & 1.51 & 19.0 \\
\hline Kwabedu (KW 1) & Borehole & 5.93 & 1.1 & 0.78 & 145 & 0.06 & 4.45 & 8.14 & 0.60 & 1.0 & $<0.1$ & 0.43 & 405 \\
\hline Asaman Kakraba (1) & Borehole & 6.20 & 0.47 & 0.12 & 268 & 0.25 & 1.17 & 1.82 & 1.96 & 4.0 & $<0.1$ & 0.41 & 302 \\
\hline Tarkwa Banso (1) & Borehole & 5.71 & 1.2 & 0.94 & 3.7 & 0.07 & 9.55 & 10.8 & 0.24 & 1.0 & $<0.1$ & 0.42 & 35.9 \\
\hline Boboobo (1) & Borehole & 5.15 & 0.13 & 0.49 & 386 & 7.6 & 4.56 & 16.2 & 2.66 & 0.5 & $<0.1$ & 1.35 & 51.3 \\
\hline Tamso & Borehole & 4.02 & 0.21 & 0.64 & 324 & 6.9 & 3.72 & 11.4 & 2.43 & 1.0 & $<0.1$ & 0.50 & 25.0 \\
\hline Manaekrom & Borehole & 5.45 & 0.10 & 1.8 & 156 & 1.2 & 0.93 & 9.72 & 1.29 & 0.5 & $<0.1$ & 2.63 & 33.4 \\
\hline Mean & & 5.60 & 1.0 & 0.78 & 797 & 2.5 & 4.29 & 10.3 & 2.90 & 1.3 & & 1.21 & 291.04 \\
\hline Median & & 5.71 & 0.77 & 0.57 & 296 & 0.90 & 3.90 & 8.93 & 1.23 & 1.0 & & 1.10 & 67.32 \\
\hline Kamproase Down Below & Well & 4.55 & 0.08 & 0.31 & 80.7 & 1.3 & 0.87 & 18.5 & 0.44 & $<0.1$ & $<0.1$ & 0.34 & 5.5 \\
\hline Behind IRS & Well & 5.66 & 0.66 & 0.66 & 275 & 0.43 & 4.09 & 42.3 & 2.28 & 1.0 & $<0.1$ & 4.19 & 38.7 \\
\hline Tarkwa Nabooso & Well & 5.56 & 0.11 & 0.19 & 232 & 3.8 & 5.10 & 19.0 & 2.26 & $<0.1$ & $<0.1$ & 14.0 & 64.7 \\
\hline John Tabiri's Residence & Well & 4.76 & 0.07 & 0.29 & 43.8 & 0.31 & 1.30 & 31.3 & 0.70 & $<0.1$ & $<0.1$ & 0.71 & 7.6 \\
\hline Government Hospital & Well & 5.33 & 0.10 & 0.26 & 108 & 0.50 & 1.63 & 35.2 & 1.95 & $<0.1$ & $<0.1$ & 1.65 & 27.0 \\
\hline New Site & Well & 4.49 & 0.06 & 0.13 & 264 & 1.2 & 5.19 & 10.6 & 4.72 & $<0.1$ & $<0.1$ & 3.26 & 33.5 \\
\hline Mean & & 5.06 & 0.18 & 0.30 & 167 & 1.2 & 3.03 & 26.2 & 2.06 & & & 4.02 & 29.5 \\
\hline Median & & 5.04 & 0.09 & 0.27 & 170 & 0.85 & 2.86 & 25.1 & 2.11 & & & 2.45 & 30.2 \\
\hline Efuanta (ES1) & Stream & 6.40 & 0.30 & 0.33 & 658 & 0.50 & 2.36 & 11.7 & 1.24 & 2.0 & $<0.1$ & 6.41 & 90.1 \\
\hline Nsuta & Stream & 6.32 & 0.24 & 0.39 & 1590 & 0.90 & 1.45 & 7.22 & 1.12 & 5.0 & $<0.1$ & 5.83 & 60.5 \\
\hline Atuabo & Stream & 5.82 & 0.24 & 0.45 & 895 & 6.9 & 2.91 & 10.4 & 2.02 & 63 & $<0.1$ & 2.94 & 36.6 \\
\hline Asomdwee (AS1) & Stream & 5.82 & 0.22 & 0.42 & 318 & 0.86 & 1.51 & 10.5 & 0.62 & 8.0 & $<0.1$ & 3.30 & 22.6 \\
\hline Kamproase (KS) & Stream & 6.47 & 0.22 & 0.28 & 475 & 0.55 & 1.48 & 1495 & 1.22 & 2.0 & $<0.1$ & 4.58 & 76.1 \\
\hline Asaman Kakraba (AK) & Stream & 6.47 & 0.14 & 0.23 & 35.4 & 0.05 & 0.97 & 4.05 & 1.41 & 0.5 & $<0.1$ & 2.09 & 50.4 \\
\hline Kwamenakrom & Stream & 6.27 & 0.32 & 0.77 & 2530 & 0.78 & 2.95 & 80.5 & 1.00 & 14 & $<0.1$ & 15.5 & 76.8 \\
\hline Tarkwa Goldfields (1) & Stream & 7.80 & 0.02 & 0.10 & 11.1 & 0.08 & 1.87 & 3.43 & 1.51 & 8.0 & $<0.1$ & 1.93 & 578 \\
\hline Tarkwa Goldfields (2) & Stream & 7.85 & 0.05 & 0.28 & 18.6 & 0.15 & 3.51 & 4.39 & 3.17 & 10 & $<0.1$ & 3.81 & 1240 \\
\hline River Anwanafo Akrase & River & 6.24 & 0.61 & 0.93 & 440 & 2.9 & 9.17 & 10.7 & 2.14 & 21 & $<0.1$ & 3.25 & 32.3 \\
\hline River Bonsa & River & 6.40 & 2.0 & 1.2 & 929 & 1.2 & 1.97 & 7.15 & 0.57 & 4.0 & $<0.1$ & 3.15 & 46.7 \\
\hline Tarkwa Bans Abiri & River & 7.74 & 1.1 & 0.81 & 283 & 0.47 & 1.67 & 14.5 & 0.46 & 73 & $<0.1$ & 2.97 & 262 \\
\hline Mean & & 6.63 & 0.46 & 0.52 & 682 & 1.3 & 2.65 & 138 & 1.37 & 18 & & 4.65 & 214 \\
\hline Median & & 6.40 & 0.24 & 0.41 & 457 & 0.67 & 1.92 & 10.5 & 1.23 & 8.0 & & 3.27 & 68.3 \\
\hline Control Sample & Tap & 7.5 & 0.08 & 0.08 & 3.3 & 0.01 & 1.76 & 3.22 & 1.84 & $<0.1$ & $<0.1$ & 1.59 & 39.3 \\
\hline
\end{tabular}

TABLE 1 cont'd

Source

Type Mo Ag

Cd In Sn

$\mathbf{S b}$

Cs

Ba

$\mathrm{Hg}$

Tl

$\mathbf{P b}$ 


\begin{tabular}{|c|c|c|c|c|c|c|c|c|c|c|c|c|c|}
\hline Tarkwa School of Mines & Borehole & 0.18 & 0.15 & 0.15 & 0.01 & 0.02 & $<0.1$ & $<0.1$ & 1.6 & $<0.5$ & 0.01 & 0.21 & 0.02 \\
\hline Tarkwa Secondary School (1) & Borehole & 2.19 & 0.15 & 0.16 & 0.13 & 0.31 & 0.2 & 0.2 & 520 & $<0.5$ & 0.14 & 0.32 & 0.14 \\
\hline Tarkwa Secondary School (2) & Borehole & 1.79 & 0.09 & 0.03 & 0.02 & 0.04 & $<0.1$ & 0.1 & 23 & $<0.5$ & 0.02 & 0.24 & 0.02 \\
\hline Akoon (1) & Borehole & 0.13 & 0.10 & 0.09 & $<0.01$ & $<0.01$ & $<0.1$ & 0.4 & 35 & $<0.5$ & $<0.01$ & 0.01 & 0.00 \\
\hline Akoon (2) & Borehole & 0.36 & 0.45 & 0.03 & 0.01 & 0.05 & $<0.1$ & 0.2 & 28 & 2.3 & 0.01 & 0.25 & 0.01 \\
\hline Atuabo & Borehole & 0.13 & 0.17 & 0.06 & 0.04 & 0.12 & 0.1 & 0.1 & 20 & $<0.5$ & 0.05 & 0.25 & 0.04 \\
\hline Kwabedu (KW 1) & Borehole & 0.08 & 0.28 & 0.07 & 0.02 & 0.06 & $<0.1$ & 0.1 & 13 & $<0.5$ & 0.02 & 0.20 & 0.02 \\
\hline Asaman Kakraba (1) & Borehole & 0.14 & 0.13 & 0.02 & 0.01 & 0.02 & $<0.1$ & 0.3 & 43 & $<0.5$ & 0.02 & 0.09 & 0.01 \\
\hline Tarkwa Banso (1) & Borehole & 0.08 & 0.03 & 0.01 & $<0.01$ & 0.01 & $<0.1$ & $<0.1$ & 5.1 & $<0.5$ & $<0.01$ & 0.18 & 0.01 \\
\hline Boboobo (1) & Borehole & 0.23 & 0.11 & 0.05 & $<0.01$ & $<0.01$ & $<0.1$ & 0.1 & 57 & $<0.5$ & $<0.01$ & 0.02 & $<0.01$ \\
\hline Tamso & Borehole & 0.06 & 0.09 & 0.06 & $<0.01$ & 0.01 & $<0.1$ & $<0.1$ & 52 & $<0.5$ & $<0.01$ & 0.08 & 0.01 \\
\hline Manaekrom & Borehole & 0.05 & 0.03 & 0.03 & 0.02 & 0.02 & $<0.1$ & 0.1 & 28 & $<0.5$ & 0.03 & 0.06 & 0.02 \\
\hline Mean & & 0.45 & 0.15 & 0.06 & 0.03 & 0.07 & & 0.2 & 69 & & 0.04 & 0.16 & 0.03 \\
\hline Median & & 0.14 & 0.12 & 0.06 & 0.02 & $\mathbf{0 . 0 3}$ & & 0.1 & 28 & & 0.02 & 0.19 & 0.02 \\
\hline Kamproase Down Below & Well & 0.04 & 0.14 & 0.05 & 0.01 & 0.02 & $<0.1$ & $<0.1$ & 9.3 & $<0.5$ & 0.01 & 0.43 & 0.01 \\
\hline Behind IRS & Well & 0.09 & 0.18 & 0.08 & $<0.01$ & 0.12 & 0.2 & 0.1 & 49 & $<0.5$ & 0.03 & 43 & 0.01 \\
\hline Tarkwa Nabooso & Well & 0.05 & 0.16 & 0.07 & $<0.01$ & 0.02 & $<0.1$ & 0.1 & 55 & $<0.5$ & 0.03 & 0.30 & $<0.01$ \\
\hline John Tabiri's Residence & Well & 0.05 & 0.17 & 0.04 & $<0.01$ & 0.06 & 0.1 & $<0.1$ & 15 & $<0.5$ & 0.01 & 0.67 & $<0.01$ \\
\hline Government Hospital & Well & 0.09 & 0.29 & 0.06 & $<0.01$ & 0.17 & $<0.1$ & $<0.1$ & 43 & 1 & 0.02 & 1.04 & $<0.01$ \\
\hline New Site & Well & 0.04 & 0.14 & 0.06 & $<0.01$ & 0.01 & $<0.1$ & $<0.1$ & 110 & $<0.5$ & 0.04 & 1.27 & $<0.01$ \\
\hline Mean & & 0.06 & 0.18 & 0.06 & & 0.07 & & & 47 & & 0.02 & 7.76 & \\
\hline Median & & 0.05 & 0.17 & 0.06 & & 0.04 & & & 46 & & 0.02 & 0.86 & \\
\hline Efuanta (ES1) & Stream & 0.56 & 0.28 & 0.13 & $<0.01$ & 0.03 & 0.1 & $<0.1$ & 27 & $<0.5$ & $<0.01$ & 1.09 & 0.01 \\
\hline Nsuta & Stream & 6.50 & 0.09 & 0.01 & $<0.01$ & 0.01 & 0.2 & $<0.1$ & 25 & $<0.5$ & $<0.01$ & 0.32 & $<0.01$ \\
\hline Atuabo & Stream & 0.29 & 0.15 & 0.03 & $<0.01$ & 0.03 & 0.2 & $<0.1$ & 44 & $<0.5$ & 0.01 & 0.72 & 0.01 \\
\hline Asomdwee (AS1) & Stream & 0.13 & 0.15 & 0.00 & $<0.01$ & 0.02 & $<0.1$ & $<0.1$ & 13 & $<0.5$ & $<0.01$ & 0.28 & $<0.01$ \\
\hline Kamproase (KS) & Stream & 0.45 & 0.13 & 0.05 & 0.01 & 0.02 & $<0.1$ & $<0.1$ & 26 & $<0.5$ & 0.01 & 2.01 & 0.01 \\
\hline Asaman Kakraba (AK) & Stream & 0.08 & 0.02 & 0.00 & $<0.01$ & 0.01 & $<0.1$ & $<0.1$ & 31 & $<0.5$ & $<0.01$ & 0.16 & $<0.01$ \\
\hline Kwamenakrom & Stream & 1.57 & 0.12 & 0.08 & $<0.01$ & 0.05 & 0.1 & 0.1 & 21 & $<0.5$ & $<0.01$ & 1.01 & $<0.01$ \\
\hline Tarkwa Goldfields (1) & Stream & 0.72 & 0.04 & 0.00 & $<0.01$ & $<0.01$ & $<0.1$ & 0.3 & 36 & $<0.5$ & $<0.01$ & 0.04 & $<0.01$ \\
\hline Tarkwa Goldfields (2) & Stream & 1.54 & 0.04 & 0.00 & $<0.01$ & $<0.01$ & 0.1 & 0.8 & 75 & $<0.5$ & $<0.01$ & 0.02 & $<0.01$ \\
\hline River Anwanafo Akrase & River & 0.73 & 1.35 & 0.03 & 0.01 & 0.08 & 0.5 & $<0.1$ & 44 & $<0.5$ & 0.01 & 3.71 & 0.04 \\
\hline River Bonsa & River & 1.19 & 0.09 & 0.02 & $<0.01$ & 0.01 & 0.1 & $<0.1$ & 12 & $<0.5$ & $<0.01$ & 0.45 & $<0.01$ \\
\hline Tarkwa Bans Abiri & River & 223 & 0.09 & 0.12 & $<0.01$ & 0.02 & 15 & 0.1 & 9.6 & $<0.5$ & $<0.01$ & 0.42 & $<0.01$ \\
\hline Mean & & 19.7 & 0.21 & 0.04 & & $\mathbf{0 . 0 3}$ & 2.0 & & 30 & & & 0.85 & \\
\hline Median & & 0.73 & 0.10 & 0.03 & & 0.02 & 0.1 & & 27 & & & 0.43 & \\
\hline Control Sample & Tap & 0.25 & 0.01 & $<0.01$ & $<0.01$ & 0.01 & $<0.1$ & $<0.1$ & 38 & $<0.5$ & $<0.01$ & 0.05 & $<0.01$ \\
\hline
\end{tabular}

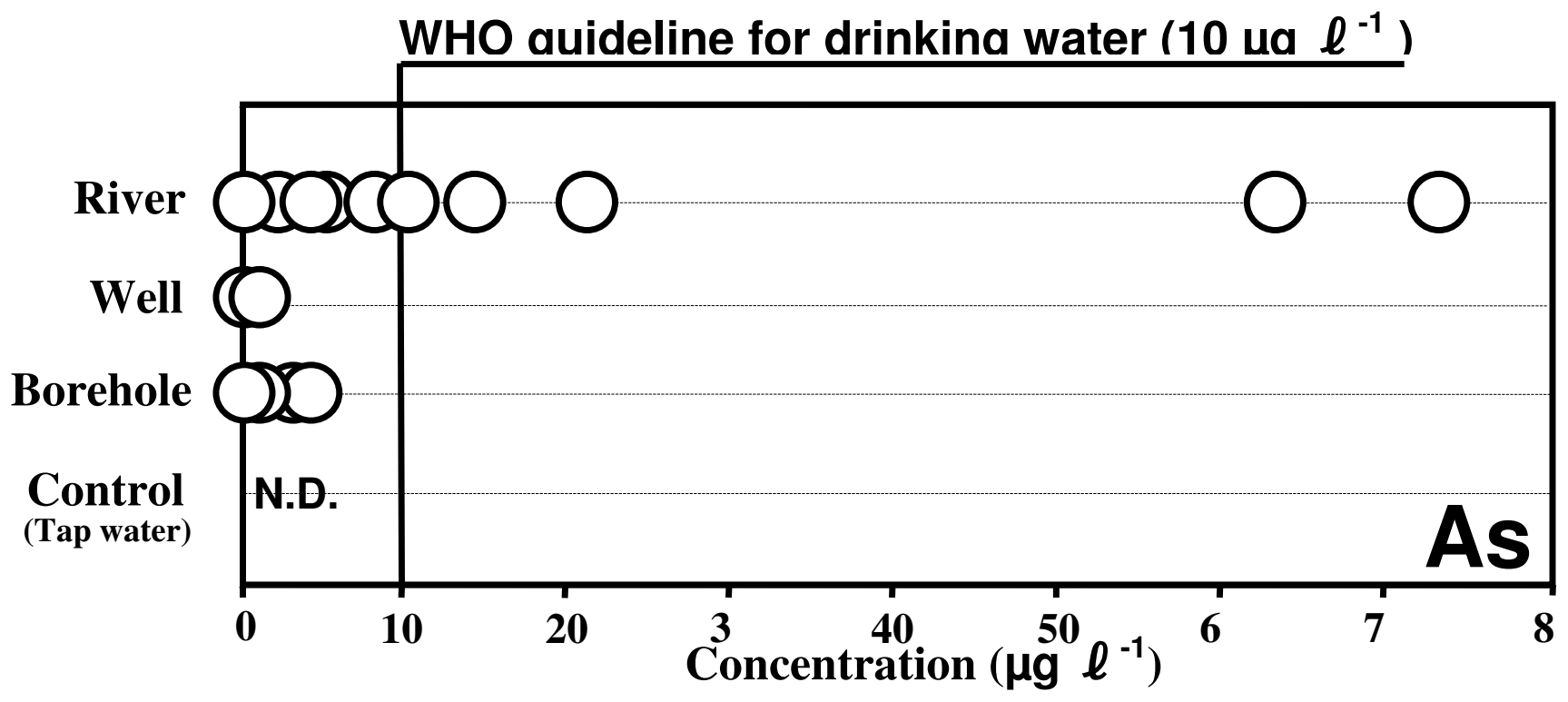


The relatively high levels of arsenic in river waters suggest that people living in this area might be at risk. The highest As concentration exceeded the highest levels in some studies from literature for either ground or river water;14.4 $\mathrm{g} \mathrm{g}^{1^{-1}}$ (Tamasi \& Cini, 2004), $22 \mu \mathrm{g} \mathrm{l}^{-1}$ (Rios-Arana et al., 2003), $13.8 \mu \mathrm{g} \mathrm{l}^{-1}$ (Naicker et al., 2003), $21 \mu \mathrm{g}$ $\mathrm{l}^{-1}$ (Miller et al., 2004), $49.3 \mu \mathrm{g} \mathrm{l}^{-1}$ (Meza et al., 2004) and $64 \mu \mathrm{g} \mathrm{l}^{-1}$ (Smedley, 1996) but were much less than concentrations observed in regions, where chronic health problems related to As, have been detected; $<0.01-2.5$ $\mathrm{mg} \mathrm{l}^{-1}$ (Anawar et al., 2002), < 1 - $535 \mu \mathrm{g} \mathrm{l}^{-1}$ (Watanabe et al., 2001), $3.05 \mu \mathrm{g} \mathrm{l^{-1 }}$ (Berg et al., 2001) and 1,654 $\mathrm{gg} \mathrm{l}^{-1}$ (Chakraborti et al., 2003). In the present study, relatively high As concentrations in the river waters may reflect the oxidation/weathering of arsenopyrite and, probably, other sulphide-bearing mine tailings.

Mercury was detected in only two of the water samples, and one of them from a borehole $\left(2.3 \mu \mathrm{g} \mathrm{l}^{-1}\right)$ exceeded the WHO guideline level of $1 \mu \mathrm{g} \mathrm{l^{-1 }}$ for $\mathrm{Hg}$ in drinking water (WHO, 2004). In a related study carried out in a smallscale gold mining town of Dumasi in Ghana, $\mathrm{Hg}$ concentrations in boreholes varied from 0.12 to $0.27 \mu \mathrm{g} \mathrm{l^{-1 }}(\mathrm{n}=8)$ and concentrations in river water samples were more variable, ranging between 0.18 and $0.76 \mu \mathrm{g} \mathrm{l}^{-1}(\mathrm{n}=5)($ Babut et al., 2003). Although $\mathrm{Hg}$ contamination by gold mining is also of concern in Tarkwa, the results suggest that, currently, it is not so serious. However, the study might have failed to detect some $\mathrm{Hg}$ contamination because the detection limit $\left(<0.5 \mu \mathrm{g} \mathrm{l}^{-1}\right)$ for $\mathrm{Hg}$ in this study was higher than those in other studies (Babut et al., 2003; Golow \& Mingle, 2003). The highest lead concentra-tion of $43 \mu \mathrm{g} \mathrm{l}^{-1}$ was measured from a well. This well is located in a commercial area, and lead-contaminated street dust or residential soil and lead-based paint from the buildings in the vicinity could have contributed to the elevated value (Banerjee, 2003; Montgomery \& Mathee, 2005). The rest of the samples were all below the WHO guideline value of $10 \mu \mathrm{g} \mathrm{l}^{-1}$ for drinking water (WHO, 2004).

Manganese is also an essential element but a known mutagen (Beckman et al., 1985). The accumulation of Mn may cause hepatic encephalopathy. Moreover, the chronic ingestion of $\mathrm{Mn}$ in drinking water is associated with neurological damage (Kondakis et al., 1989). Interestingly, about 33\% of the boreholes and 58\% of the river waters exceeded the WHO guideline value of $400 \mu \mathrm{g} \mathrm{l}^{-1}$ for Mn in drinking water (WHO, 2004) (Fig. 3). Thus, a health risk to the people of Tarkwa consuming Mn-contami-nated water is of great concern. The geology of the Tarkwa area consists of Tarkwaian and Birimian systems (Kuma \& Younger, 2001) and manganese-bearing horizons are quite ubiquitous in rocks of the Birimian suite.

Antimony is a well-known toxic element at high doses (Navas-Acien et al., 2005). It shares similar chemical and toxicological properties with arsenic (Gebel, 1997), and they are frequent co-exposures (Gebel et al., 1998). Antimony in drinking water has been reported to modulate the toxicity of arsenic (Gebel, 1999), and the severity of As poisoning might be magnified by exposure to Sb (Frisbie et al., 2002). Incidentally, the same sample that recorded the highest As concentration of $73 \mu \mathrm{g} \mathrm{l}^{-1}$ had the highest Sb concentration of $15 \mu \mathrm{g} \mathrm{l}^{-1}$, exceeding the WHO drinking water guideline value of $5 \mu \mathrm{g} \mathrm{l}^{-1}$ for Sb. The rest of the samples were below the detection limit of $0.1 \mu \mathrm{g}{ }^{-1}$. The concentrations of the other trace elements in the water samples were generally low with most concentrations below the limits of detection (Table 1). Arsenic was not detected in the control sample and the concentrations of the other trace elements in the control sample were predominantly low and within the WHO safe limits for drinking water (Table 1).

\section{Urine samples}

Urinary As concentrations from the study area varied between 34 and $700 \mu \mathrm{g} \mathrm{l}^{-1}$ with a mean of $260 \mu \mathrm{g} \mathrm{l^{-1 }}$ (Table 2). The highest values of $700 \mu \mathrm{g} \mathrm{l}^{-1}$ and $650 \mu \mathrm{g} \mathrm{l}^{-1}$ were from a female (unknown occupation) and a male mine worker, respectively. The urinary As levels of the control samples ranged from 120 to $280 \mu \mathrm{g} \mathrm{l}^{-1}$ (mean value of 200 $\left.\mu \mathrm{g} \mathrm{l}^{-1}\right)$. In terms of sex, the mean values for males and females were 250 and $260 \mu \mathrm{g} \mathrm{l}^{-1}$, respectively. Statistically, there was no difference between males and females $(P=0.876)$. There was also no correlation between urinary As concentration and age of males and females $(r=0.056)$ (Fig. 4). In this study, the youngest female (21 years) and oldest female (51 years) had urinary As levels of $290 \mu \mathrm{g} \mathrm{l}^{-1}$ and $43 \mu \mathrm{g} \mathrm{l}^{-1}$, respectively. The onset of menopause may add to the explanation of the change of urinary As levels in women (Kristiansen et al., 1997). The distribution of 
urinary As concentrations among the miners and non-miners (from the study area) and control samples is shown in Fig. 5 and, statistically, there was no difference among the three groups.

TABLE 2

Trace element concentrations $(\mu \mathrm{g} / \mathrm{l})$ in urine samples from the study area (Tarkwa) and control site (Accra)

\begin{tabular}{|c|c|c|c|c|c|c|c|c|c|c|c|c|c|c|}
\hline Subject & Occupation & \multicolumn{2}{|c|}{ Age (yrs) } & Sex & $V$ & $\mathrm{Cr}$ & $M n$ & Co & $\mathrm{Cu}$ & $Z n$ & $G a$ & As & $\mathrm{Se}$ & $R b$ \\
\hline $\mathrm{T} 1$ & Mine worker & 30 & Male & 1.3 & 11 & 4.5 & 1.0 & 263 & 146 & 0.10 & 85 & 23 & 207 & 69.7 \\
\hline $\mathrm{T} 2$ & Mine worker & 29 & Male & 2.0 & 12 & 13.3 & 1.5 & 503 & 620 & 0.61 & 380 & 99 & 3250 & 383 \\
\hline $\mathrm{T} 3$ & Mine worker & 59 & Male & 1.0 & 11 & 4.5 & 0.8 & 244 & 64.1 & $<0.01$ & 34 & 8 & 1170 & 21.0 \\
\hline T5 & Mine worker & 45 & Male & 2.8 & 21 & 3.8 & 1.0 & 367 & 699 & 0.21 & 330 & 48 & 1720 & 229 \\
\hline T6 & Mine worker & 29 & Male & 4.1 & 27 & 5.0 & 1.7 & 648 & 1230 & 0.21 & 650 & 58 & 2270 & 292 \\
\hline $\mathrm{T} 7$ & Mine worker & 41 & Male & 4.4 & 24 & 7.4 & 1.7 & 615 & 681 & 0.42 & 230 & 68 & 1280 & 545 \\
\hline $\mathrm{T} 10$ & Mine worker & 42 & Male & 4.9 & 27 & 4.6 & 2.3 & 516 & 220 & 0.21 & 170 & 38 & 2190 & 210 \\
\hline T11 & Mine worker & 26 & Male & 2.3 & 15 & 2.6 & 1.2 & 174 & 393 & 0.20 & 160 & 21 & 520 & 397 \\
\hline $\mathrm{T} 12$ & Mine worker & 36 & Male & 2.8 & 15 & 7.8 & 1.7 & 417 & 317 & 0.10 & 140 & 80 & 2250 & 162 \\
\hline $\mathrm{T} 13$ & Mine worker & 29 & Male & 6.0 & 25 & 8.1 & 1.8 & 349 & 928 & 0.10 & 260 & 74 & 6790 & 87.6 \\
\hline T14 & Mine worker & 43 & Male & 6.2 & 34 & 12.3 & 1.7 & 421 & 279 & 0.21 & 400 & 270 & 8120 & 224 \\
\hline $\mathrm{T} 15$ & Mine worker & 45 & Female & 4.8 & 29 & 25.1 & 1.4 & 388 & 801 & 0.20 & 260 & 70 & 6380 & 93.1 \\
\hline $\mathrm{T} 21$ & Cleaner & 34 & Male & 2.8 & 17 & 6.4 & 1.3 & 163 & 2020 & 0.10 & 430 & 111 & 7310 & 192 \\
\hline $\mathrm{T} 22$ & Military & 25 & Male & 2.1 & 18 & 5.6 & 0.8 & 132 & 495 & 0.10 & 130 & 61 & 2020 & 44.1 \\
\hline $\mathrm{T} 23$ & Unknown & 26 & Male & 3.1 & 19 & 5.2 & 1.3 & 221 & 1100 & 0.10 & 270 & 71 & 7830 & 112 \\
\hline $\mathrm{T} 24$ & Unknown & 34 & Female & 6.4 & 29 & 6.4 & 2.1 & 580 & 159 & 0.21 & 230 & 62 & 3350 & 273 \\
\hline $\mathrm{T} 25$ & Unknown & 20 & Female & 3.0 & 23 & 6.2 & 2.4 & 272 & 958 & 0.10 & 60.0 & 18 & 6100 & 155 \\
\hline $\mathrm{T} 26$ & Unknown & 25 & Female & 2.6 & 19 & 103 & 2.9 & 358 & 628 & 0.52 & 700 & 123 & 9660 & 15.6 \\
\hline $\mathrm{T} 27$ & Unknown & 19 & Male & 4.8 & 22 & 8.0 & 3.6 & 528 & 695 & 0.20 & 150 & 76 & 6320 & 192 \\
\hline T28 & Unknown & 43 & Female & 3.8 & 22 & 9.3 & 2.3 & 516 & 770 & 0.31 & 285 & 56 & 2400 & 342 \\
\hline T29 & Unknown & 26 & Male & 2.7 & 17 & 8.3 & 1.1 & 489 & 294 & 0.10 & 70 & 43 & 3580 & 103 \\
\hline T30 & Unknown & 32 & Female & 4.1 & 21 & 42.7 & 3.3 & 377 & 850 & 0.41 & 300 & 52 & 1680 & 382 \\
\hline T31 & Unknown & 27 & Female & 19 & 28 & 262 & 3.2 & 476 & 719 & 5.65 & 310 & 122 & 6350 & 135 \\
\hline T32 & Unknown & 21 & Female & 2.6 & 22 & 5.3 & 4.1 & 490 & 1600 & 0.31 & 290 & 85 & 2270 & 534 \\
\hline Median & & 26 & & 4.9 & 24 & 4.2 & 1.8 & 614 & 598 & 0.10 & 200 & 73 & 2290 & 263 \\
\hline
\end{tabular}

TABLE 2 cont'd

\begin{tabular}{|c|c|c|c|c|c|c|c|c|c|c|c|c|c|c|c|}
\hline Subject & Occupation & Age & (yrs) & Sex & Mo & $A g$ & $C d$ & In & $S n$ & $S b$ & Cs & $B a$ & $\mathrm{Hg}$ & $T l$ & $P b$ \\
\hline $\mathrm{T} 1$ & Mine worker & 30 & Male & 18.2 & $<0.01$ & 0.10 & 0.03 & 2.4 & 0.1 & 5 & 0.8 & 1.3 & 0.30 & 2.0 & $<0.01$ \\
\hline $\mathrm{T} 2$ & Mine worker & 29 & Male & 258 & 0.82 & 0.72 & 0.04 & 3.1 & 0.3 & 8 & 9.3 & 7.1 & 0.41 & 6.1 & 0.05 \\
\hline $\mathrm{T} 3$ & Mine worker & 59 & Male & 10.0 & 0.20 & 0.10 & 0.01 & 6.1 & 0.1 & 1 & 0.5 & 0.5 & 0.10 & 1.0 & $<0.01$ \\
\hline $\mathrm{T} 4$ & Mine worker & 40 & Male & 146 & 0.51 & 0.92 & 0.03 & 2.3 & 0.2 & 14 & 3.5 & 4.5 & 0.41 & 8.2 & 0.01 \\
\hline T5 & Mine worker & 45 & Male & 134 & $<0.01$ & 0.72 & 0.03 & 2.2 & 0.2 & 5 & 0.9 & 2.4 & 0.41 & 5.1 & $<0.01$ \\
\hline T6 & Mine worker & 29 & Male & 78.1 & $<0.01$ & 0.82 & $<0.01$ & 0.9 & 0.1 & 8 & 2.2 & 1.4 & 0.21 & 2.1 & $<0.01$ \\
\hline $\mathrm{T} 7$ & Mine worker & 41 & Male & 60.2 & 1.4 & 0.63 & 0.11 & 1.1 & 0.2 & 6 & 5.4 & 9.4 & 0.42 & 5.3 & 0.08 \\
\hline T8 & Mine worker & 41 & Male & 147 & 0.21 & 0.62 & 0.03 & 2.3 & 0.1 & 8 & 1.5 & 3.5 & 0.21 & 6.2 & 0.01 \\
\hline
\end{tabular}




\begin{tabular}{|c|c|c|c|c|c|c|c|c|c|c|c|c|c|c|c|}
\hline T9 & Mine worker & 48 & Male & 149 & 0.31 & 0.92 & 0.3 & 3.1 & 0.4 & 9 & 1.6 & 4.3 & 0.61 & 10.2 & 0.20 \\
\hline $\mathrm{T} 10$ & Mine worker & 42 & Male & 82.2 & 0.10 & 0.52 & 0.01 & 1.5 & 0.1 & 6 & 1.4 & 2.4 & 0.21 & 2.1 & $<0.01$ \\
\hline $\mathrm{T} 11$ & Mine worker & 26 & Male & 34.2 & 0.20 & 0.10 & $<0.01$ & 1.0 & 0.1 & 3 & 4.0 & 2.8 & 0.10 & 4.0 & $<0.01$ \\
\hline T12 & Mine worker & 36 & Male & 31.9 & 0.62 & 0.62 & 0.04 & 2.0 & 0.2 & 6 & 1.4 & 2.0 & 0.31 & 2.1 & 0.03 \\
\hline T13 & Mine worker & 29 & Male & 60.8 & 0.41 & 0.41 & 0.03 & 1.1 & 0.1 & 10 & 0.8 & 3.5 & 0.52 & 5.2 & 0.01 \\
\hline T14 & Mine worker & 43 & Male & 29.9 & 0.83 & 1.03 & 0.04 & 1.5 & 0.3 & 12 & 1.2 & 3.0 & 0.62 & 7.2 & 0.01 \\
\hline T15 & Mine worker & 45 & Female & 36.8 & 0.82 & 0.41 & 0.1 & 4.8 & 0.2 & 6 & 3.5 & 5.9 & 0.51 & 3.1 & 0.02 \\
\hline T16 & Food vendor & 30 & Female & 43.9 & 0.92 & 0.92 & 0.01 & 1.0 & 0.1 & 5 & 1.4 & 2.3 & 0.20 & 2.0 & 0.02 \\
\hline $\mathrm{T} 17$ & Caterer & 24 & Male & 140 & 0.83 & 0.73 & 0.02 & 0.9 & 0.2 & 12 & 3.2 & 4.9 & 0.83 & 4.1 & 0.03 \\
\hline T18 & Trader & 31 & Female & 49.2 & 1.5 & 0.31 & 0.04 & 2.1 & 0.1 & 6 & 13 & 3.6 & 0.31 & 3.1 & 0.01 \\
\hline T19 & Nurse & 44 & Male & 38.8 & 0.75 & 0.43 & 0.03 & 0.6 & 0.1 & 7 & 1.6 & 3.2 & 0.32 & 3.2 & 0.02 \\
\hline $\mathrm{T} 20$ & Nurse & 51 & Female & 25.3 & 0.51 & 0.20 & 0.03 & 0.9 & 0.1 & 1 & 1.1 & 1.1 & 0.10 & 1.0 & 0.02 \\
\hline $\mathrm{T} 21$ & Cleaner & 34 & Male & 42.5 & 0.41 & 0.52 & 0.02 & 0.8 & 0.2 & 13 & 0.9 & 12 & 0.52 & 7.3 & 0.03 \\
\hline $\mathrm{T} 22$ & Military & 25 & Male & 153 & 0.92 & 1.03 & 0.02 & 0.6 & 0.1 & 5 & 0.6 & 2.7 & 0.21 & 2.1 & 0.02 \\
\hline $\mathrm{T} 23$ & Unknown & 26 & Male & 90.9 & 0.51 & 0.61 & 0.02 & 1.4 & 0.2 & 11 & 0.7 & 4.5 & 0.31 & 3.1 & 0.03 \\
\hline $\mathrm{T} 24$ & Unknown & 34 & Female & 57.8 & 0.62 & 0.83 & 0.03 & 1.8 & 0.1 & 6 & 4.1 & 7.6 & 0.21 & 2.1 & 0.03 \\
\hline $\mathrm{T} 25$ & Unknown & 20 & Female & 39.1 & 0.62 & 0.10 & 0.02 & 1.4 & 0.2 & 7 & 0.6 & 1.1 & 0.41 & 4.1 & 0.08 \\
\hline $\mathrm{T} 26$ & Unknown & 25 & Female & 141 & 0.42 & 0.83 & 0.04 & 1.6 & 0.2 & 22 & 8.9 & 8.2 & 0.63 & 6.3 & 0.03 \\
\hline $\mathrm{T} 27$ & Unknown & 19 & Male & 40.9 & 0.51 & 0.31 & 0.04 & 1.1 & 0.1 & 7 & 3.5 & 4.0 & 0.41 & 3.1 & 0.01 \\
\hline $\mathrm{T} 28$ & Unknown & 43 & Female & 74.0 & 1.0 & 0.82 & 0.02 & 0.8 & 0.1 & 7 & 2.8 & 2.8 & 0.21 & 2.1 & 0.02 \\
\hline T29 & Unknown & 26 & Male & 89.6 & 0.10 & 0.31 & $<0.01$ & 1.6 & 0.1 & 7 & 1.9 & 3.4 & 0.21 & 4.1 & 0.01 \\
\hline T30 & Unknown & 32 & Female & 102 & 0.20 & 0.72 & 0.01 & 3.7 & 0.2 & 6 & 6.7 & 2.5 & 0.20 & 7.2 & $<0.01$ \\
\hline T31 & Unknown & 27 & Female & 69.0 & 0.42 & 1.9 & 0.27 & 2.8 & 1 & 10 & 86 & 5.2 & 0.63 & $<0.01$ & 0.26 \\
\hline T32 & Unknown & 21 & Female & 14.2 & 0.10 & 0.61 & 0.01 & 1.5 & 0.2 & 9 & 3.7 & 4.6 & 0.31 & 7.1 & 0.01 \\
\hline Mean & 34 & & 77.7 & 0.58 & 0.62 & 0.05 & 1.9 & 0.2 & 8 & 5.6 & 4.0 & 0.35 & 4.2 & 0.04 & \\
\hline Median & 32 & & 60.5 & 0.51 & 0.62 & 0.03 & 1.5 & 0.2 & 7 & 1.7 & 3.4 & 0.31 & 4.0 & 0.02 & \\
\hline \multicolumn{16}{|c|}{ CONTROL } \\
\hline $\mathrm{C} 1$ & Lab. Technician & 26 & Male & 71.4 & $<0.01$ & 0.31 & $<0.01$ & 1.8 & 0.1 & 6 & 0.8 & 5.5 & 0.21 & 1.0 & $<0.01$ \\
\hline $\mathrm{C} 2$ & Lab. Technician & 26 & Male & 59.0 & $<0.01$ & 0.41 & $<0.01$ & 2.0 & 0.2 & 4 & 2.8 & 2.0 & 0.10 & 1.0 & $<0.01$ \\
\hline $\mathrm{C} 3$ & Lab. Technician & 25 & Male & 107 & $<0.01$ & 0.31 & $<0.01$ & 1.1 & 0.1 & 5 & 1.9 & 3.5 & 0.21 & 2.1 & $<0.01$ \\
\hline $\mathrm{C} 4$ & Lab. Technician & 27 & Male & 328 & $<0.01$ & 0.42 & $<0.01$ & 1.2 & 0.1 & 7 & 1.5 & 1.4 & 0.31 & 3.1 & $<0.01$ \\
\hline Mean & & 26 & & 141 & & 0.36 & & 1.5 & 0.1 & 6 & 1.7 & 3.1 & 0.21 & 1.8 & \\
\hline Median & & 26 & & 89.1 & & 0.36 & & 1.5 & 0.1 & 6 & 1.7 & 2.8 & 0.21 & 1.6 & \\
\hline
\end{tabular}

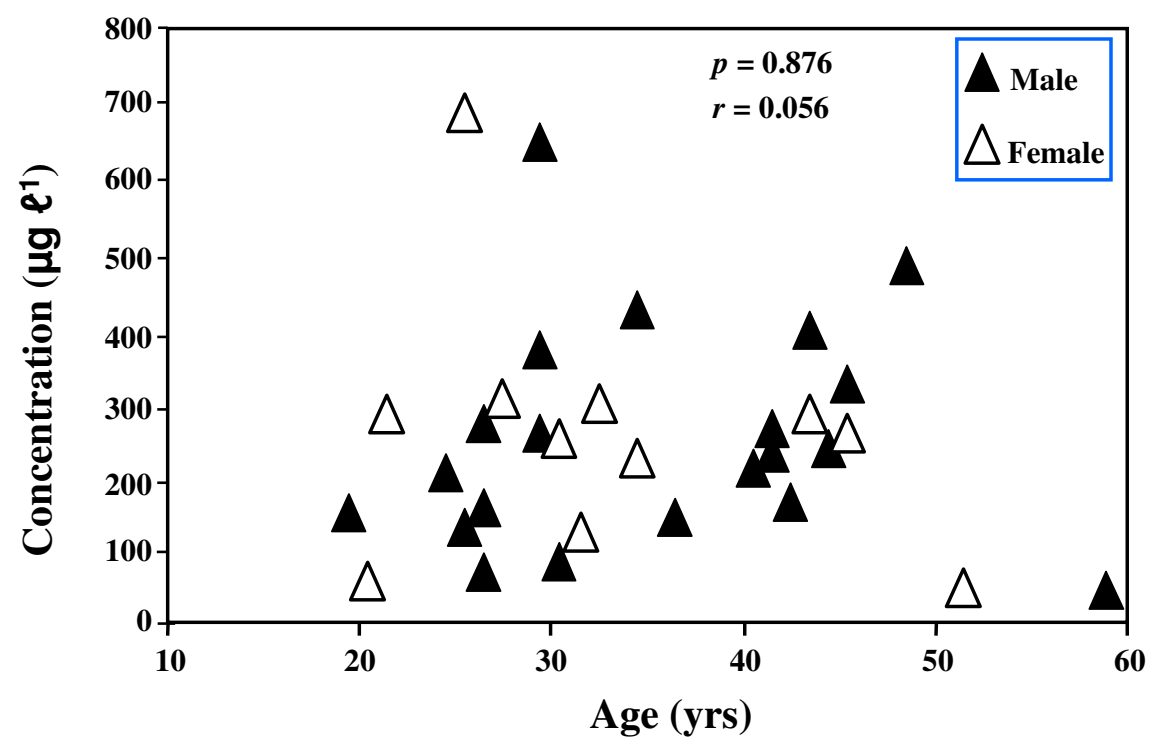

Figure 4. Correlation between urinary arsenic concentration and age of males and females. 


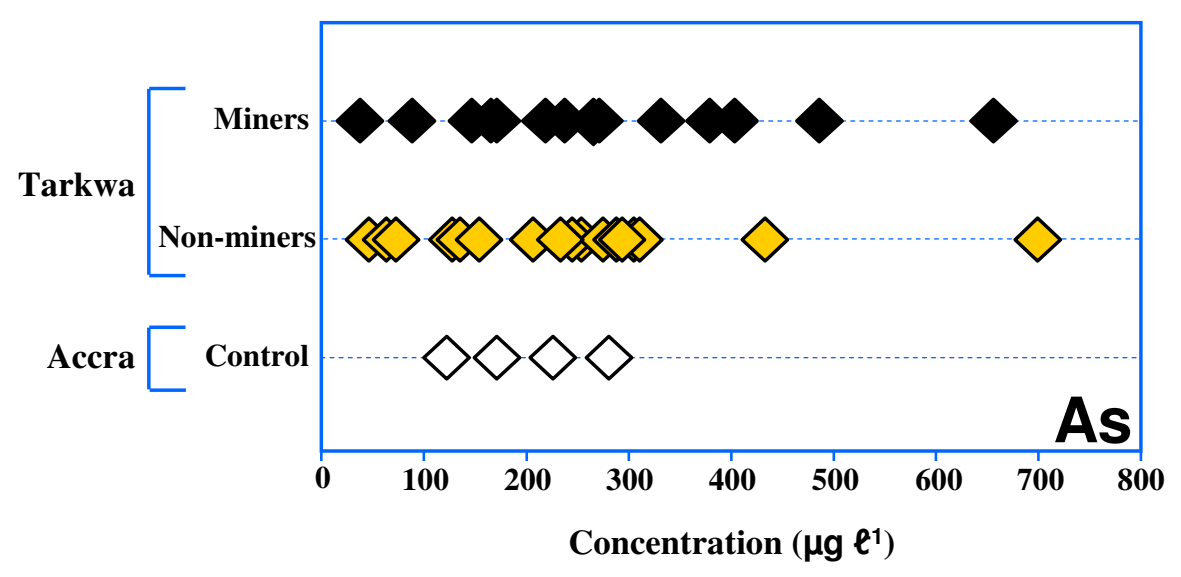

Figure 5. Urinary arsenic concentrations in miners, non-miners and control subjects.

The mean urinary As concentration of $260 \mu \mathrm{g} \mathrm{l}^{-1}$ was higher than values from a study of 22 people from arsenicaffected villages in West Bengal, India (Samanta et al., 2000). In the West Bengal study, mean urinary As concentrations of $170.4 \mu \mathrm{gl}^{-1}$ (adult males), $169 \mu \mathrm{g} 1^{-1}$ (adult females) and $151.9 \mu \mathrm{g}^{-1}$ (children) were reported (Samanta et al., 2000). Similarly, the mean urinary As concentration from this study was higher than other studies that involved communities depending on tube wells and other water sources with diverse arsenic concentrations for drinking and cooking purposes. Concentrations measured were 18.9-93.8 $\mu \mathrm{g} 1^{-1}$ (Meza et al., 2004), 1.6-40 $\mu \mathrm{g} 1^{-1}$ (Kristiansen et al., 1997) and 0.7-19.4 $\mu \mathrm{g} 1^{-1}$ (White et al., 1998). However, the range of concentration in this study 34-700 $\mu \mathrm{g} 1^{-1}$, was much lower than that of Semria Ojha Patti village 24-3,696 $\mu \mathrm{g} 1^{-1}$ in West Bengal, India, and Samta village 110-3,085 $\mu \mathrm{g} 1^{-1}$ in Bangladesh (Chakraborti et al., 2003). Nevertheless, the results indicate relatively high degree of human exposure to arsenic in Tarkwa, Ghana, when compared to some well-known heavily arsenicaffected countries of the world (Fig. 6). 


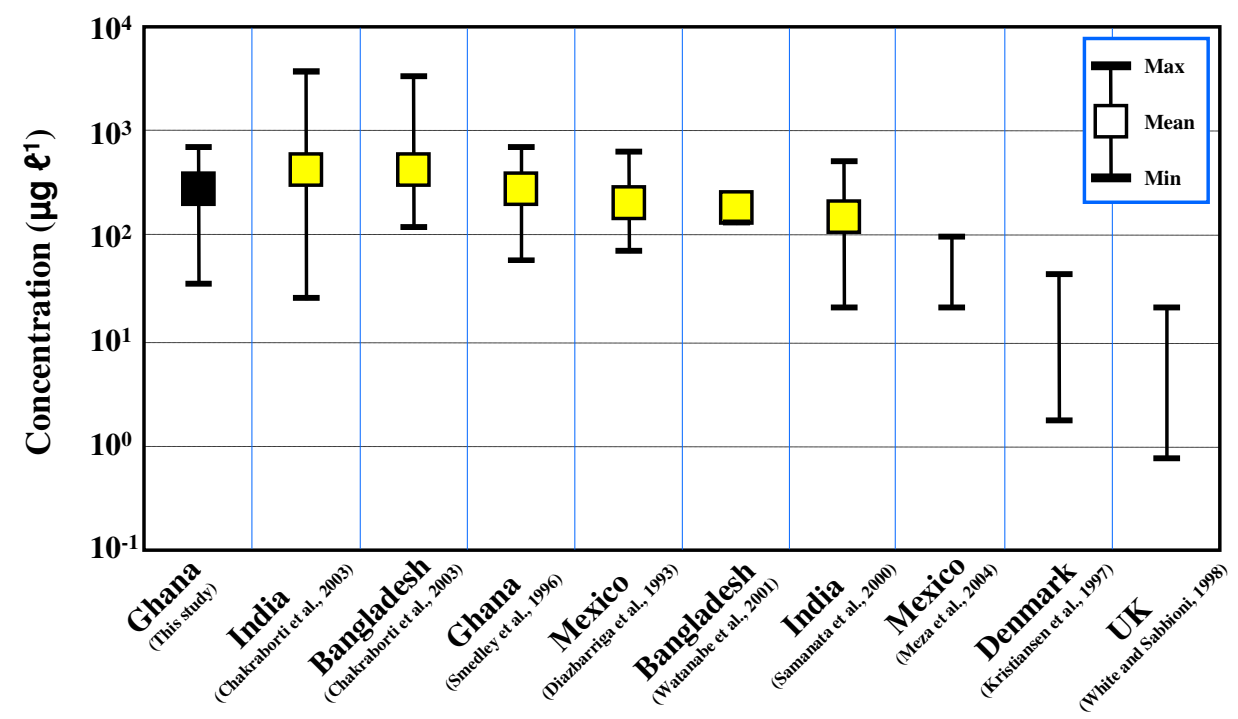

Figure 6. Comparison of urinary arsenic concentrations from this study with other countries.

Factors, such as how often fish is consumed, time of alcohol consumption, number of amalgam fillings, as well as working hours are probably important to the evaluation of $\mathrm{Hg}$ contamination of people occupationally exposed to $\mathrm{Hg}$ vapours (Santa Rosa et al., 2001). In this study, $\mathrm{Hg}$ concentrations ranged from $<0.5-12 \mu \mathrm{g} \mathrm{l}^{-1}$ with a mean of 4 $\mu \mathrm{g} \mathrm{l}^{-1}$. The range in this present study fell below the range of $0.02-183 \mu \mathrm{g} \mathrm{l}^{-1}$ reported in a study involving four towns around the Ankobra and Tano river basins in south-western Ghana of which the 217 subjects (12-18 years) were of school going age and might be involved in illegal mining 'galamsey' activities (Adimado \& Baah, 2002). Van Straaten (2000) also reported $\mathrm{Hg}$ concentration of $172 \mathrm{mg} \mathrm{l}^{-1}$ in a 29-year old male involved in small-scale gold mining in Tanzania. The reason for the low $\mathrm{Hg}$ levels in the present study could be that the subjects were not into small-scale gold mining which involves amalgamation of the gold concentrate and later heating the amalgam to vapourise $\mathrm{Hg}$.

In this study, concentrations of Cd varied between 0.10 and $1.9 \mu \mathrm{g} \mathrm{l}^{-1}$ (mean of $0.62 \mu \mathrm{g} \mathrm{l}^{-1}$ ), and fell below the urinary Cd concentration of $20 \mu \mathrm{g} \mathrm{l}^{-1}$, generally considered as normal (Horng, 1996). The concentrations of urinary $\mathrm{Cr}$ in this study ranged from $9.4 \mathrm{~g} \mathrm{l}^{-1}$ to $34 \mathrm{~g} \mathrm{l}^{-1}$ with a mean value of $21 \mu \mathrm{g} \mathrm{l}^{-1}$. The range was significantly higher than some reference studies: 0.04-1.5 $\mu \mathrm{g} \mathrm{l}^{-1}$ (Minoia et al., 1990), 0.1-0.52 $\mathrm{gg} \mathrm{l}^{-1}$ (Brune et al., 1993) and $<0.7 \mu \mathrm{g} \mathrm{l^{-1 }}$ (Paschal et al., 1998). It must, however, be emphasized that the subjects in this present study were quite different from the reference studies in that samples were taken mainly from patients who were likely to be exposed.

Copper concentrations in this study ranged from 72.7 to $676 \mu \mathrm{g} \mathrm{l}^{-1}$ and levels of $\mathrm{Zn}$ varied from 64.1 to $2020 \mu \mathrm{g} \mathrm{\textrm {l } ^ { - }}$ ${ }^{1}$. Some reported urinary $\mathrm{Cu}$ concentrations are 12-80 $\mu \mathrm{g} \mathrm{l}^{-1}$ (Iyengar \& Woittiez, 1988) and 4.2-50 $\mu \mathrm{g} \mathrm{1^{-1 }}$ (Minoia et al., 1990) while that for urinary $\mathrm{Zn}$ are 180-850 $\mu \mathrm{g} \mathrm{l}^{-1}$ (Schramel et al., 1985), 266-864 $\mu \mathrm{g} \mathrm{l}^{-1}$ (Minoia et al., 1990) and 361-599 $\mu \mathrm{g} \mathrm{l}^{-1}$ (Iyengar \& Woittiez, 1988). Higher concentrations of $\mathrm{Cu}$ were observed in the control site compared with the study area, suggesting the existence of some exposure sources in the control site. Since the samples, especially from control site $(n=4)$, were limited, further study is required to confirm the regional difference of trace element levels. For urinary trace elements, the pattern of elemental abundance was $\mathrm{Rb}>\mathrm{Zn}>\mathrm{Cu}$ $>\mathrm{As}>\mathrm{Sr}>\mathrm{Mo}>\mathrm{Se}>\mathrm{Cr}>\mathrm{Mn}>\mathrm{Cs}>\mathrm{Ba}$. On the other hand, concentrations of $\mathrm{Sb}$, In and Bi were low or below detection limits. Fig. 7 shows the comparison of urinary trace element concentrations between miners and control subjects. 

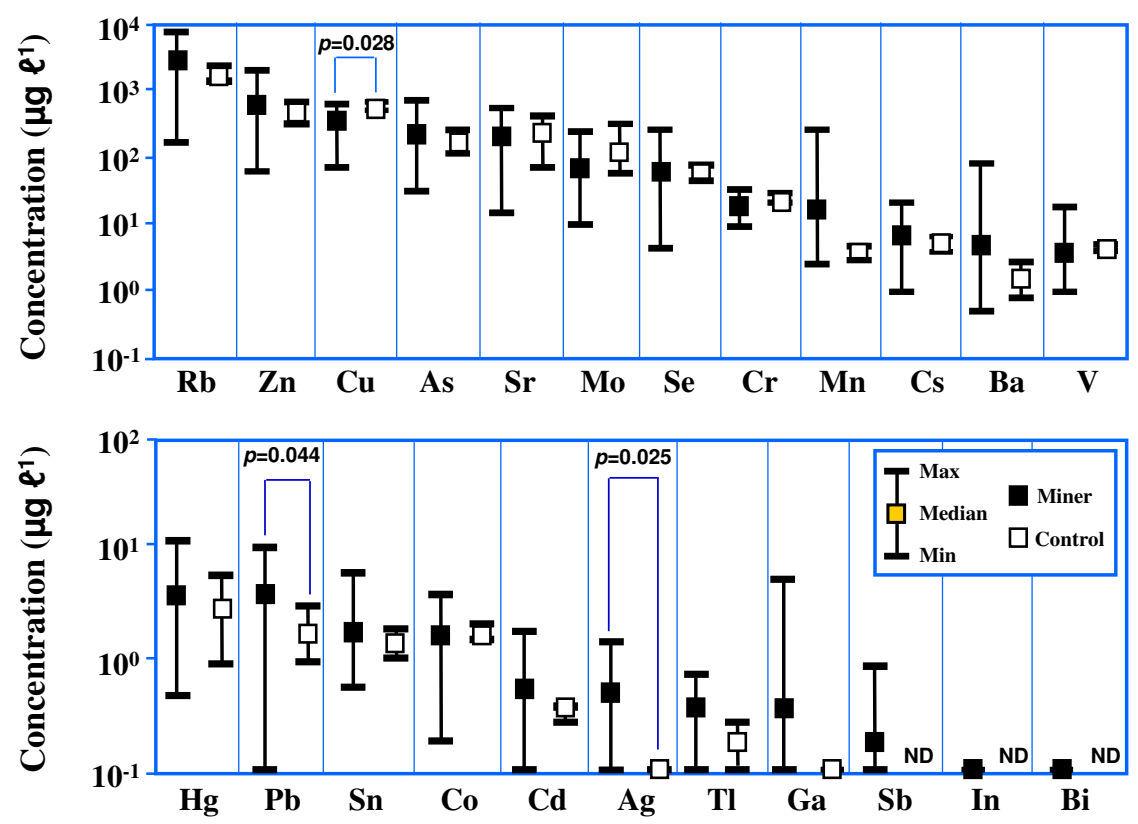

Figure 7. Comparison of urinary trace element concentrations between miners and control subjects.

Relationships between concentrations of As in water and human urine samples

In an attempt to assess the arsenic contamination in Ghana and identify the possible exposure route, a graph of arsenic concentration in urine against arsenic level in water from some well-known arsenic-affected countries of the world was plotted (Fig. 8). Consumption of groundwater is an important exposure route of As to residents in Ascontaminated regions such as Bangladesh and India (West Bengal). For Bangladesh, India (West Bengal), Chile and Mexico, as the concentrations of arsenic in water increased the corresponding levels in urine also increased significantly $(r=0.651, P=0.022)$, implying that the source of As in urine could be from drinking water. Both studies in China were conducted in the Guizhou Province, an area of endemic arsenicosis, where a unique type of exposure resulting from the burning of As-rich coal is found. Guizhou inhabitants commonly use this coal for cooking, heating, and drying their dietary staples of corn and hot peppers. 


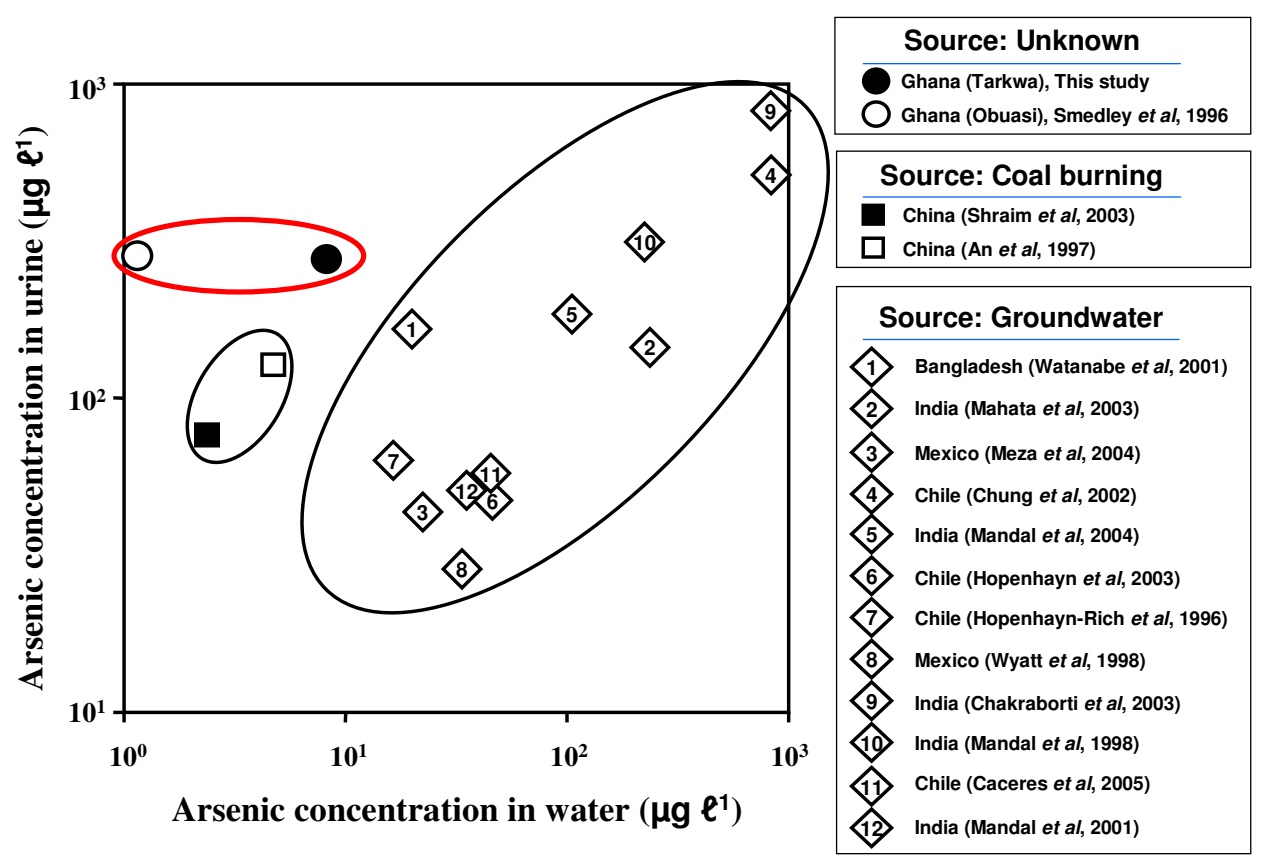

Figure 8. Relationships between concentrations of As in water and human urine in different populations.

The coal is burned in open stoves without chimneys, resulting in contamination of both the indoor air and the foods being prepared. In this present study, although the arsenic levels in water samples were much lower than those in other highly As-endemic regions, the concentrations in human urine were comparable (Fig. 8). A similar trend was also observed in an earlier study in the Obuasi area of Ghana by Smedley et al. (1996) who analyzed As concentrations in urine and groundwater, and suggested that the high levels of total As in urine and low levels of total As in drinking water reflected high fish intake. However, since they did not analyze arsenobetaine (AB) and metabo-lized inorganic arsenicals such as monome-thylarsonic acid (MMA) and dimethylarsinic acid (DMA) (Fig. 9) in urine samples, fish may not always be a significant source of As exposure. 


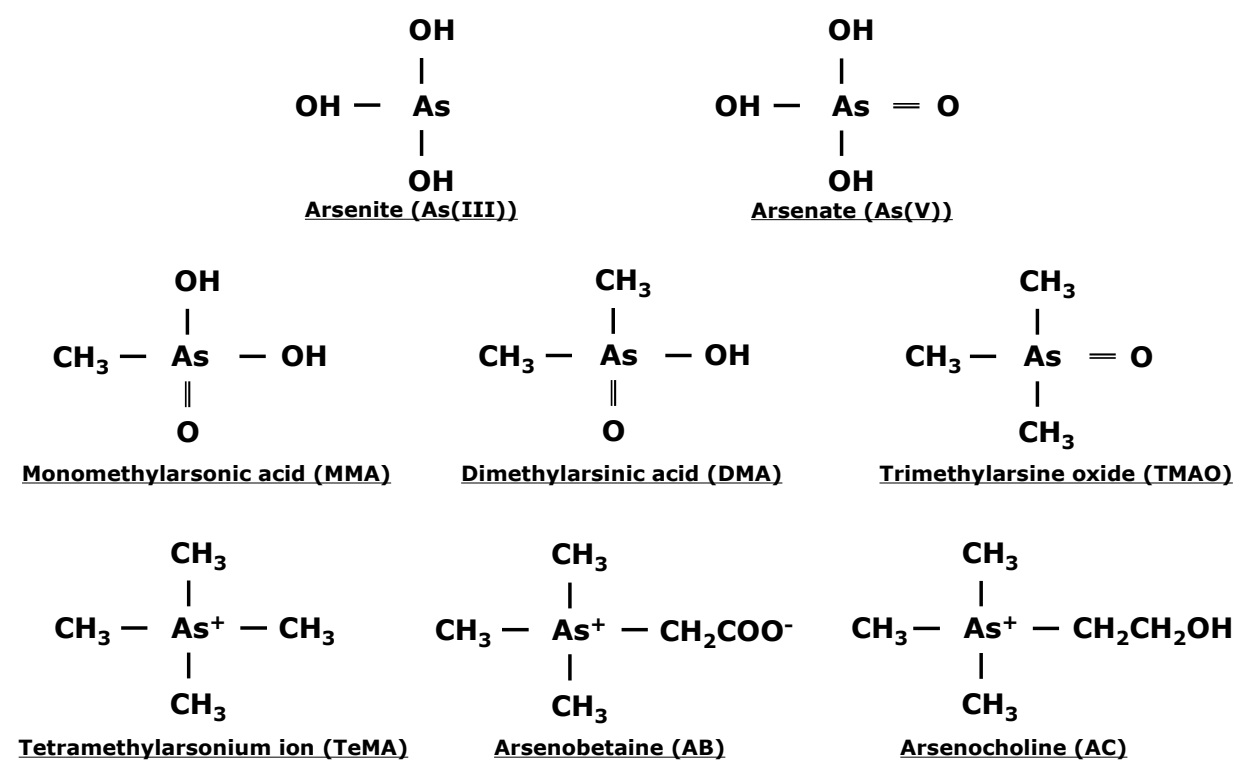

Figure 9. Chemical structures of arsenic species.

Some foodstuffs are known to contain high concentrations of As. Seafood consumption may contribute intakes of As because seafood has high As concentrations and As is generally present as organic species such as arsenobetaine (Cullen \& Reimer, 1989), and, to a lesser extent, arsenocholine, both of which are of very low toxicity. Arsenobetaine, which is ingested through the marine products, is rapidly excreted into urine without change (Le et al., 1993). Thus, total As in urine measured in this study may not be an appropriate measure of inorganic As exposure because of the dietary artifacts associated with certain types of seafood containing arsenobetaine, arsenocholine and arseno-sugar (organoarsenic compounds), which are excreted through urine without metabolic transformation. When total urinary As is measured, intake of all forms of arsenic is detected. Organic As is, however, consi-dered relatively low-toxic.

\section{Conclusion}

Unlike other areas of the developing world, e.g. Bangladesh, West Bengal in India, southern Taiwan, Chile, Vietnam, Argentina and Mexico, where endemic As poisoning is prevalent, groundwaters from the study area are apparently less vulnerable to the generation of high dissolved As concentra-tions. Primary sulphide minerals could have been leached and As present in the system strongly bound to ferric oxide products rather than being significantly mobilized into solution. Nevertheless, based on the results of this study, $\mathrm{As}, \mathrm{Mn}, \mathrm{Pb}$ and $\mathrm{Hg}$ contamination in some water sources is a cause for concern. Other elements that showed high urinary concentrations relative to the control samples are $\mathrm{Pb}, \mathrm{Ag}, \mathrm{Mn}, \mathrm{Rb}, \mathrm{Zn}, \mathrm{Cd}, \mathrm{Ba}, \mathrm{Tl}, \mathrm{Sb}$ and $\mathrm{Ga}$.

Tarkwa is an important case study for assessing the health effects of As and other trace elements because it depicts a typical mining town. Urinary As concentrations were comparable to those in some well-known arsenicaffected countries of the world. Relatively low As levels in water and no significant difference in urinary As concentrations between the study area and control site suggest other sources of As contamination in Ghana, possibly food.

The health of the population within the area surveyed as well as the economic resources are threatened by As contamina-tion and other trace elements arising from mining activities. If the geologic and groundwater conditions that promote high As concentrations are known, they may be helpful to identify high-risks areas. Social conscience about health risks and consequences of environmental pollution has to be developed and the actual situation must be taken into account by authorities (Environmental Protection Agency, Minerals Commission, Ghana Chamber of 
Mines, etc.) to achieve a definite solution to the problem. For example, the powers of the district assemblies should be enhanced to monitor activities and enforce regulations on polluting discharges into the environment from mining companies and also check the illegal activities of small-scale miners.

Arsenic is a serious health threat as exposure to this element, particularly in the inorganic form, can lead to cancer. Recent studies have shown that As acts as an endocrine disruptor at very low concentra-tion (Kaltreider $e t$ al., 2001). Total As was determined in this study. In future, an attempt should be made to determine the different species of As in drinking water, human urine, and fish and food items to evaluate the intake of inorganic As in Ghana. Since the most common As species in water are inorganic, this represents potentially the most detrimental source of As in human diet. Arsenic analysis in human hair and nails may also be interesting as it depicts long term accumulation.

\section{Acknowledgement}

The authors are grateful to Messrs Sampson K. Abu and Kwame Danso of CSIR-WRI, and the Tarkwa Government Hospital and Aba Hospital, for their help in sampling. They also acknowledge Mr Masahiro Kunimoto and Miss Noriko Tsunehiro, staff in the Environmental Specimen Bank (es-BANK), CMES, Ehime University, Japan, for their support in sample management. This study was supported by 21st Century COE Program from the Ministry of Education, Culture, Sports, Science and Technology, Japan.

\section{References}

Adimado A. A. and Baah D. A. (2002). Mercury in human blood, urine, hair, nail and fish from the Ankobra and Tano River Basins in Southwestern Ghana. Bull. Envir. Contam. Tox. 68: 339-346.

Agusa T., Kunito T., Fujihara J., Kubota R., Minh T. B., Kim Trang P. T., Iwata H., Subramanian A., Viet P. H. and Tanabe S. (2006). Contamination by arsenic and other trace elements in tube-well water and its risk assessment to humans in Hanoi, Vietnam. Envir. Pollut. 139: 95-106.

Akabzaa T. (2000). Boom and Dislocation: The environmental and social impacts of mining in the Wassa West District of Ghana. Third World Network-Africa. pp. 1-131.

Alessio L. and Foa V. (1983). Lead. In Human Biological Monitoring of Industrial Chemicals Series, vol. 1. (L. Alessio, A. Berlin, R. Roi and M. Boni, ed.), pp. 105-132. CEC Joint Research Centre, Ispra Establishment, Ispra, Italy.

Amasa S. K. (1975). Arsenic pollution at Obuasi goldmine, town and surrounding countryside. Envir. Hlth Perspect. 12: $131-135$.

Amonoo-Neizer E. H. (1980). Arsenate adsorption by some Ghana soils. Bull. Sci. Ass. Niger. 6(1):2.

Amonoo-Neizer E. H. and Amekor E. M. K. (1993). Determination of total arsenic in environmental samples from Kumasi and Obuasi, Ghana. Envir. Hlth Perspect. 101(1): 44-49.

Amonoo-Neizer E. H. and Busari G. L. (1980). Arsenic status of Ghana soils: Contamination of soils near a gold smelter. Ghana J. Sci. 20 $(1,2): 57-62$.

An D., He Y. G. and Hu Q. X. (1997). Poisoning by coal smoke containing arsenic and fluoride. Fluoride 30: 29-32.

Anawar H. M., Akai J., Mostofa K. M. G., Safiullah S. and Tareq S. M. (2002). Arsenic poisoning in groundwater: Health risk and geochemical sources in Bangladesh. Envir. Int. 27: 597-604.

Babut M., Sekyi R., Rambaud A., Potin-Gautier M., Tellier S., Bannerman W. and Beinhoff C. (2003). Improving the environmental management of small-scale gold mining in Ghana: a case study of Dumasi. J. Clean Prod. 11: 215-221.

Banerjee A. D. K. (2003). Heavy metal levels and solid phase speciation in street dust. Envir. Pollut. 123: 95-105.

Beckman R. A., Milvran A. S. and Loeb L. A. (1985). On the fidelity of DNA replication: manganese mutagenesis in vitro. Biochemistry 24: $5810-5817$.

Berg M., Tran H. C., Nguyen T. C., Pham H. V., Schertenleib R. and Giger W. (2001). Arsenic contamination of groundwater and drinking water in Vietnam: a human health threat. Envir. Sci. Technol. 35: 2621-2626.

Braman R. S. and Foreback C. C. (1973). Methylated forms of arsenic in the environment. Science 182: 1247-1249.

Brune D., Atitio A., Nordberg G., Vesteber G. O. and Gerhardsson L. (1993). Normal concentration of chromium in serum and urine a TRACY project. Scand. J. Wk Envir. Hlth 1: 39-44.

Caceres D. D., Pino P., Montesinos N., Atalah E., Amigo H. and Loomis D. (2005). Exposure to inorganic arsenic in drinking water and total urinary arsenic concentration in a Chilean population. Envir. Res. 98: 151-159.

Chakraborti D., Mukherjee S. C., Pati S., Sengupta M. K., Rahman M. M., Chowdhury U. K., Lodh D., Chanda C. R., Chakraborti A. K. and Basu G. K. (2003). Arsenic groundwater contamination in Middle Ganga Plain, Bihar, India: A future danger? Envir. Hlth Perspect. 111 (9): 1194-1201.

Chung J. S., Kalman D. A., Moore L. E., Kosnett M. J., Arroyo A. P., Beeris M., Mazumder D. N. G., Hernandez A. L. and Smith A. H. (2002). Family correlations of arsenic methylation patterns in children and parents exposed to high concentrations of arsenic in drinking water. Envir. Hlth Perspect. 110 (7): 729-733.

Cullen W. R. and Reimer K. J. (1989). Arsenic speciation in the environment. Chem. Rev. 89: 713-764. 
Diazbarriga F., Santos M. A., Mejia J. J., Batres L., Yanez L., Carrizales L., Vera E., Del Razo L. M. and Cebrian M. E. (1993). Arsenic and cadmium exposure in children living near a smelter complex in San Luis Potosi, Mexico. Envir. Res. 62: 242-250.

Frisbie S. H., Ortega R., Maynard D. M. and Sarkar B. (2002). The concentrations of arsenic and other toxic elements in Bangladesh's drinking water. Envir. Hlth Perspect. 110: 1147-1153.

Gebel T. (1997). Arsenic and antimony: comparative approach on mechanistic toxicology. Chem. Biol. Interact. 107 (3): $131-144$.

Gebel T. W. (1999). Arsenic and drinking water contamination. Science 283: 1458-1459.

Gebel T. W., Suchenwirth R. H., Bolten C. and Dunkelberg H. H. (1998) Human biomonitoring of arsenic and antimony in case of an elevated geogenic exposure. Envir. Hlth Perspect. 106: 33-39.

Golow A. A., Schlueter S., Amihere-Mensah S., Granson H. L. K. and Tetteh M. S. (1996). Distribution of arsenic and sulphate in the vicinity of Ashanti Goldmine at Obuasi, Ghana. Bull. Envir. Contam. Toxicol. 56: 703-710.

Golow A. A. and Adzei E. A. (2002). Mercury in surface soil and cassava crop near an alluvial goldmine at Dunkwa-on-Offin, Ghana. Bull. Envir. Contam. Toxicol. 69: 228-235.

Golow A. A. and Mingle L. C. (2003). Mercury in river water and sediments in some rivers near Dunkwa-On-Offin, an Alluvial Goldmine, Ghana. Bull. Envir. Contam. Toxicol. 70: 379-384.

Hopenhayn-Rich C., Biggs M. L., Smith A. H., Kalman D. A. and Moore L. E. (1996). Methylation study of a population environmentally exposed to arsenic in drinking water. Envir. Hlth Perspect. 104: 620-627.

Hopenhayn C., Huang B., Christian J., Peralta C., Ferreccio C., Atallah R. and Kalman D. (2003). Profile of urinary arsenic metabolites during pregnancy. Envir. Hlth Perspect. 111(16): 1888-1891.

Horng C-J. (1996). Simultaneous determination of urinary $\mathrm{Zn}, \mathrm{Cd}, \mathrm{Pb}$ and $\mathrm{Cu}$ concentration in steel production workers by differential pulse anodic stripping voltammetry. Analyst 121: 1511-1514.

Horng C-J., Tsai J-L., Horng P-H., Lin S-C., Lin S-R. and Tzeng C-C. (2002). Determination of urinary lead, cadmium and nickel in steel production workers. Talanta 56: 1109-1115.

Hsueh Y. M., Huang Y. L., Huang C. C., Wu W. L., Chen H. M., Yang M. H., Lue L. C. and Chen C. J. (1998). Urinary levels of inorganic and organic arsenic metabolites among residents in arsenic-hyperendemic area in Taiwan. J. Toxicol. Envir. Hlth 54 (6): $431-$ 444.

Iyengar G. V. and Woittiez J. (1988). Trace elements in human clinical specimens: evaluation of literature data to identify reference values. Clin. Chem. 34: 474-481.

Kaltreider R. C., Davis A. M., Lariviere J. P. and Hamilton J. W. (2001). Arsenic alters the function of the glucocorticoid receptor as a transcription factor. Envir. Hlth Perspect. 109: 245-251.

Kondakis X. G., Makris N., Leotsinidis M., Prino M. and Papapetropoulos T. (1989). Possible health effects of high manganese concentration in drinking water. Arch. Envir. Hlth 44(3): 175-178.

Kristiansen J., Christensen J. M., Iversen B. S. and Sabbioni E. (1997). Toxic trace element concentrations in blood and urine: influence of gender and lifestyle factors. Sci. tot. Envir. 204: 147-160.

Kuma J. S. and Younger P. L. (2001). Pedological characteristics related to groundwater occurrence in the Tarkwa area, Ghana. J. Afr. Earth Sci. 33(2): 363-376.

Lagerkvist B. E. A., Linderholm H. and Nordberg G. F. (1988). Arsenic and Raynaud's phenomenon: vasospastic tendency and excretion of arsenic in smelter workers before and after summer vacation. Int. Arch. Occup. envir. Hlth 60: 361-364.

Lai V. W-M., Sun Y., Tin E., Cullen W. R. and Reimer K. J. (2004). Arsenic speciation in human urine: are we all the same? Toxicol. Appl. Pharmacol. 198: 297-306.

Le X-C., Cullen W. R. and Reimer K. J. (1993). Determination of urinary arsenic and impact of dietary arsenic intake. Talanta 40: 185-193.

Luh M. D., Baker R. A. and Henley D. E. (1973). Arsenic analysis and toxicity - a review. Sci. tot. Envir. 2(1): 1-12.

Mahata J., Basu A., Ghoshal S., Sarkar J. N., Roy A. K., Poddar G., Nandy A. K., Banerjee A., Ray K., Natarajan A. T., Nilsson R. and Giri A. K. (2003). Chromosomal aberrations and sister chromatid exchanges in individuals exposed to arsenic through drinking water in West Bengal, India. Mutat. Res. 534: 133-143.

Mandal B. K., Chowdhury T. R., Samanta G., Mukherjee D. P., Chanda C. R., Saha K. C. and Chakraborti D. (1998). Impact of safe water for drinking and cooking on five arsenic-affected families for 2 years in West Bengal, India. Sci. tot. Envir. 218: 185-201.

Mandal B. K., Ogra Y. and Suzuki K. T. (2001). Identification of dimethylarsinous and monomethylarsinous acids in human urine of the arsenic affected areas in West Bengal, India. Chem. Res. Toxicol. 14: 371-378.

Mandal B. K., Ogra Y., Anzai K. and Suzuki K. T. (2004). Speciation of arsenic in biological samples. Toxicol. appl. Pharmacol. 198: 307-318.

Mead M. N. (2005). Arsenic: A global poison. Envir. Hlth Perspect. 113(6): 379-386.

Meza M. M., Kopplin M. J., Burgess J. L. and Gandolfi A. J. (2004). Arsenic drinking water exposure and urinary excretion among adults in the Yaqui Valley, Sonora, Mexico. Envir. Res. 96: 119-126.

Miller J. R., Hudson-Edwards K. A., Lechler P. J., Preston D. and Macklin M. G. (2004). Heavy metal contamination of water, soil and produce within riverine communities of the Rio Pilcomayo basin, Bolivia. Sci. tot. Envir. 320: 189-209.

Minoia C., Sabbioni E., Apostoli P., Pietra R., Pozzoli L., Gallorini M., Nicolaou G., Alessio L. and Capodaglio E. (1990). Trace element reference values in tissues from inhabitants of the European community. I. A study of 46 elements in urine, blood and serum of Italian subjects. Sci. tot. Envir. 95: 89-105. 
Montgomery M. and Mathee A. (2005). A preliminary study of residential paint lead concentrations in Johannesburg. Envir. Res. 98(3): 279-283.

Mushak P. and Crocetti A. F. (1995). Risk and revisionism in arsenic cancer risk assessment. Envir. Hlth Perspect. 103: 684-689.

Naicker K., Cukrowska E. and McCarthy T. S. (2003). Acid mine drainage arising from gold mining activity in Johannesburg, South Africa and environs. Envir. Pollut. 122: 29-40.

Navas-Acien A., Silbergeld E. K., Sharrett A. R., Calderon-Aranda E., Selvin E. and Guallar E. (2005). Metals in urine and peripheral arterial disease. Envir. Hlth Perspect. 113(2): 164-169.

NRC (National Research Council) (2001). Arsenic in drinking water. National Academy Press, Washington, DC.

Paschal D. C., Ting B. G., Morrow J. C., Pirkle J. L., Jackson R. J., Sampson E. J., Miller D. T. and Caldwell K. L. (1998). Trace metals in urine of United States residents: Reference range concentrations. Envir. Res. Section A 76: 53-59.

Rios-Arana J. V., Walsh E. J. and Gardea-Torresdey J. L. (2003). Assessment of arsenic and heavy metal concentrations in water and sediments of the Rio Grande at El Paso-Juarez metroplex region. Envir. Int. 29: 951-971.

Samanta G., Chowdhury U. K., Mandal B. K., Chakraborti D., Sekaran N. C., Tokunaga H. and Ando M. (2000). High performance liquid chromatography inductively coupled plasma mass spectrometry for speciation of arsenic compounds in urine. Microchem. J. 65: 113-127.

Santa Rosa R. M. S., Mueller R. C. S., Alves C. N., Sarkis J. E. S., Bentes M. H. S., Brabo E. and Oliveira E. S. (2000). Determination of total mercury in workers' urine in gold shops of Itaituba, Para State, Brazil. Sci. tot. Envir. 261: 169-176.

Schramel P., Lill G. and Hasse S. (1985). Mineral and trace elements in human urine. J. Clin. Chem. Clin. Biochem. 23: $293-301$.

Shraim A., Cui X., Li S., Ng J. C., Wang J., Jin Y., Liu Y., Guo L., Li D., Wang S., Zhang R. and Hirano S. (2003). Arsenic speciation in the urine and hair of individuals exposed to airborne arsenic through coal-burning in Guizhou, PR China. Toxicol. Lett. 137: 35-48.

Smedley P. L. (1996). Arsenic in rural groundwater in Ghana. J. Afr. Earth Sci. 22(4): 459-470.

Smedley P. L., Edmunds W. M. and Pelig-Ba K. B. (1996). Mobility of arsenic in groundwater in the Obuasi gold-mining area of Ghana: some implications for human health. Envir. Geochem. Hlth 113: 163-181.

Smedley P. L. and Kinniburgh D. G. (2002). A review of the source, behaviour and distribution of arsenic in natural waters. Appl. Geochem. 17(5): 517-568.

Tamasi G. and Cini R. (2004). Heavy metals in drinking waters from Mount Amiata (Tuscany, Italy). Possible risks from arsenic for public health in the Province of Siena. Sci. tot. Envir. 327: 41-51.

Tseng W-P. (1977). Effects and dose-response relationships of skin cancer and blackfoot disease with arsenic. Envir. Hlth Perspect. 19: 109119.

Valenzuela O. L., Borja-Aburto V. H., Garcia-Vargas G. G., Cruz-Gonzalez M. B., Garcia-Montalvo E. A., Calderon-Aranda E. S. and Del Razo L. M. (2005). Urinary trivalent methylated arsenic species in a population chronically exposed to inorganic arsenic. Envir. Hlth Perspect. 113(3): 250-254.

Vahter M. and Lind B. (1986). Concentrations of arsenic in urine of the general population in Sweden. Sci. tot. Envir. 54: 1-12.

Van Straaten P. (2000). Human mercury due to small scale gold mining in northern Tanzania. Sci. tot. Envir. 259: 45-53.

Watanabe C., Inaoka T., Kadono T., Nagano M., Nakamura S., Ushijima K., Murayama N., Miyazaki K. and Ohtsuka R. (2001). Males in rural Bangladesh communities are more susceptible to chronic arsenic poisoning than females: Analyses based on urinary arsenic. Envir. Hlth Perspect. 109(12): 1265-1270.

White M. A. and Sabbioni E. (1998). Trace element reference values in tissues from inhabitants of the European Union. X. A study of 13 elements in blood and urine of a United Kingdom population. Sci. tot. Envir. 216: 253-270.

WHO (2004). Guidelines for Drinking-water Quality, vol. 1, 3rd edn. World Health Organization, Geneva

Wyatt C. J., Quiroga V. L., Acosta R. T. O. and Mendez R. O. (1998). Excretion of arsenic (As) in urine of children, 7-11 years, exposed to elevated levels of As in the city water supply in Hermosillo, Sonora, Mexico. Envir. Res. Section A 78: 19-24. 